\title{
Local Convergence Properties of Douglas-Rachford and Alternating Direction Method of Multipliers
}

\author{
Jingwei Liang · Jalal Fadili · Gabriel Peyré
}

Received: date / Accepted: date

\begin{abstract}
The Douglas-Rachford and alternating direction method of multipliers are two proximal splitting algorithms designed to minimize the sum of two proper lower semi-continuous convex functions whose proximity operators are easy to compute. The goal of this work is to understand the local linear convergence behaviour of Douglas-Rachford (resp. alternating direction method of multipliers) when the involved functions (resp. their Legendre-Fenchel conjugates) are moreover partly smooth. More precisely, when the two functions (resp. their conjugates) are partly smooth relative to their respective smooth submanifolds, we show that Douglas-Rachford (resp. alternating direction method of multipliers) (i) identifies these manifolds in finite time; (ii) enters a local linear convergence regime. When both functions are locally polyhedral, we show that the optimal convergence radius is given in terms of the cosine of the Friedrichs angle between the tangent spaces of the identified submanifolds. Under polyhedrality of both functions, we also provide conditions sufficient for finite convergence. The obtained results are illustrated by several concrete examples and supported by numerical experiments.
\end{abstract}

Keywords Douglas-Rachford · ADMM - Partial Smoothness · Finite Activity Identification · Local Linear Convergence

Mathematics Subject Classification (2000) 49J52 - 65K05 - 65K10 • 90C25

Jingwei Liang, Jalal Fadili (Corresponding author) Normandie Univ, ENSICAEN, CNRS, GREYC, Caen, France

\{Jingwei.Liang, Jalal.Fadili\}@ensicaen.fr

Gabriel Peyré

CNRS, DMA, ENS Paris, Paris, France

Gabriel.Peyre@ens.fr 


\section{Introduction}

\subsection{Non-Smooth Optimization}

In this paper, we consider a structured optimization problem where the objective function is the sum of two proper convex and lower semi-continuous (lsc) functions on an Euclidean space. An efficient and provably convergent method to solve this optimization problem is the Douglas-Rachford (DR) splitting algorithm. DR was originally proposed in [1] to solve a system of linear equations arising from the discretization of a partial differential equation. The extension of this method suitable to solve optimization and feasibility problems is due to [2].

Global sublinear convergence rate estimates of DR and the alternating direction method of multipliers (ADMM) iterations have been recently established in the literature, see e.g. $[3,4]$ and the references therein. Such rate becomes linear under further assumptions, typically smoothness and strong convexity, see e.g. [5,6] and references therein. However, it has been observed that DR method admits local linear convergence in various situations where strong convexity is absent, and the studying of this behaviour of DR or ADMM has received increasing attentions in recent years, see the detailed discussion in Section 1.2.

Unfortunately, most of the existing work either focuses on some special cases where a specific structure of the problem at hand can be exploited, or imposes certain regularity conditions which are barely verified in practical situations. Therefore, it is important to present a unified analysis framework, and possibly with stronger claims. This is one of the main motivations of this work. More precisely, our main contributions are the following.

\section{Globally convergent non-stationary DR}

In this paper, we consider a non-stationary version of DR. By casting the non-stationarity as an additional error, in Section 4 we establish a global convergence result for the nonstationary DR iteration (6). This exploits our previous result on the convergence of the general inexact and non-stationary Krasnosel'skiı̌-Mann iteration introduced in [3].

\section{Finite time activity identification}

Assuming, that both functions in the objective are partly smooth at a global minimizer relative to smooth submanifolds (see Definition 5.1), we show in Section 5.1 that under a non-degeneracy condition, the non-stationary DR sequences respectively identify in finite time these submanifolds. In plain words, this means that after a finite number of iterations, these sequences enter these submanifolds and never leave them.

\section{Local linear convergence}

Exploiting the finite identification property, we then show that the non-stationary DR iterates converge locally linearly in Section 6 . We characterize the convergence rate precisely based on the properties of the identified partial smoothness submanifolds. Moreover, when the involved functions are locally polyhedral around a global minimizer and the DR scheme is run with constant parameters, we show that the optimal convergence rate is given in terms of the cosine of the Friedrichs angle between the 
tangent spaces of the two submanifolds. We also generalize these claims to the minimization of the sum of more than two functions.

\section{Finite convergence}

Building upon our local convergence analysis, we also characterize situations where finite convergence occurs in Section 7. More precisely, when the stationary and unrelaxed DR scheme is used and the involved functions are locally polyhedral nearby a global minimizer, and if either of the two functions is differentiable at that minimizer, we obtain finite convergence.

We also touch one some practical acceleration schemes, since once the active submanifolds are identified, the globally convex but non-smooth problem becomes locally $C^{2}$-smooth, though possibly non-convex. As a consequence, it opens the door to highorder optimization methods, such as Newton-like or nonlinear conjugate gradient.

\section{$A D M M$}

Consider the same optimization problem where, now, one of the functions is the composition of a proper lsc and convex function with an injective operator. It was shown by Gabay [7] (see also [8]), that ADMM amounts to applying DR to the FenchelRockafellar dual problem. Therefore, we can deliver the same local convergence analysis by considering the partial smoothness submanifolds of the Legendre-Fenchel conjugates of the functions in the primal problem, and that the class of partly smooth functions is closed under pre-composition by a surjective linear operator [9, Theorem 4.2]. Therefore, to avoid unnecessary repetitions, we only focus in detail on the primal DR splitting method (6).

\subsection{Relation to Prior Work}

There are problem instances in the literature where the (stationary) DR and ADMM algorithms are proved to converge linearly either globally or locally. For instance, in [2, Proposition 4], it is assumed that the "internal" function is strongly convex with a Lipschitz continuous gradient. This local linear convergence result is further investigated in $[4,6]$ under smoothness and strong convexity assumptions. The special case of Basis Pursuit (BP), i.e. one-norm minimization with an affine constraint, is considered in [10] and an eventual local linear convergence is shown in the absence of strong convexity. In [11], the author analyses the local convergence behaviour of ADMM for quadratic or linear programs, and shows local linear convergence if the optimal solution is unique and strict complementarity holds. For the case of two subspaces (though in general real Hilbert space), linear convergence of DR with the optimal rate being the cosine of the Friedrichs angle between the subspaces is proved in [12]. It turns out that $[10,11,12]$ are special cases of our framework, and our results generalize theirs to a larger class of problems. The proposed work is also a more general extension of our previous results in [13] which tackled only the case of locally polyhedral functions.

For the non-convex case, [14] considers DR for a feasibility problem of a sphere intersecting a line or more generally a proper affine subset. Such feasibility problems with an affine subspace and a super-regular set (in the sense of [15]) with strongly 
regular intersection is considered in [16], and is generalized later to two regular sets with linearly regular intersection [17], see also [18] for an even more general setting.

Our finite convergence result complements and extends that of [19] who established finite convergence of (unrelaxed stationary) DR in the presence of Slater's condition, for solving convex feasibility problems where one set is an affine subspace and the other is a polyhedron.

\subsection{Paper Organization}

The rest of the paper is organized as follows. Some preliminaries are collected in Section 2. Section 3 states our main assumptions on problem $(\mathcal{P})$ and introduces the non-stationary DR algorithm. Its global convergence is established in Section 4. Section 5 discusses the notion of partial smoothness and some essential properties. We then turn to the main contributions of this paper, namely finite time activity identification (Section 5.1), local linear convergence (Section 6) and finite termination (Section 7) of DR under partial smoothness. Section 8 extends the results to the sum of more than two functions. In Section 9, we report various numerical experiments to support our theoretical findings.

\section{Preliminaries}

Throughout the paper, $\mathbb{N}$ is the set of nonnegative integers, $\mathbb{R}^{n}$ is a $n$-dimensional real Euclidean space equipped with scalar product $\langle\cdot, \cdot\rangle$ and norm $\|\cdot\|$. Id denotes the identity operator on $\mathbb{R}^{n}$. For a vector $x \in \mathbb{R}^{n}$ and a subset of indices $b \subset\{1, \ldots, n\}$, $x_{b}$ is the restriction of $x$ to the entries indexed in $b$. Define $\|x\|_{p}:=\left(\sum_{i=1}^{p}\left|x_{i}\right|\right)^{1 / p}$, $p \in[1,+\infty]$, as the $\ell_{p}$-norm in $\mathbb{R}^{n}$ with the usual adaptation for $p=+\infty$. $\ell_{+}^{1}$ denotes the set of summable sequences in $\left[0,+\infty\left[\right.\right.$. For a matrix $M \in \mathbb{R}^{n \times n}$, we denote $\|M\|$ its operator norm and $\rho(M)$ its spectral radius.

$\Gamma_{0}\left(\mathbb{R}^{n}\right)$ is the class of proper convex and lsc functions on $\mathbb{R}^{n}$. The subdifferential of a function $J \in \Gamma_{0}\left(\mathbb{R}^{n}\right)$ is the set-valued operator,

$$
\partial J: \mathbb{R}^{n} \rightrightarrows \mathbb{R}^{n}, x \mapsto\left\{g \in \mathbb{R}^{n}: J(y) \geq J(x)+\langle g, y-x\rangle, \forall y \in \mathbb{R}^{n}\right\} .
$$

$\operatorname{prox}_{\gamma J}: \mathbb{R}^{n} \rightarrow \mathbb{R}^{n}$ denotes the proximity operator of $\gamma J$, defined as,

$$
\operatorname{prox}_{\gamma J}(\cdot):=\operatorname{argmin}_{x \in \mathbb{R}^{n}} \gamma J(x)+\frac{1}{2}\|x-\cdot\|^{2} .
$$

In the sequel, we also denote the reflected proximity operator of $\gamma J$ as

$$
\operatorname{rprox}_{\gamma J}:=2 \operatorname{prox}_{\gamma J}-\mathrm{Id} \text {. }
$$

$\operatorname{prox}_{\gamma J}$ is also the resolvent of $\gamma \partial J$, i.e. $\operatorname{prox}_{\gamma J}=(\operatorname{Id}+\gamma \partial J)^{-1}$.

For a nonempty and convex set $C \subset \mathbb{R}^{n}$, denote cone $(C)$ its conical hull, aff $(C)$ its affine hull, and the subspace parallel to $C$ is $\operatorname{par}(C)=\mathbb{R}(C-C)$, i.e. a translate of aff $(C)$ to the origin. $\mathrm{P}_{C}$ is the orthogonal projection operator onto $C$ and $N_{C}(x)$ its normal cone at $x$. 
2.1 Operators and Matrices

Definition 2.1 (Monotone operator) A set-valued operator $\mathscr{A}: \mathbb{R}^{n} \rightrightarrows \mathbb{R}^{n}$ is monotone if, given any $x, z \in \mathbb{R}^{n}$, there holds

$$
\langle x-z, u-v\rangle \geq 0, \forall(x, u) \in \operatorname{gph}(\mathscr{A}) \text { and }(z, v) \in \operatorname{gph}(\mathscr{A}) \text {, }
$$

where $\operatorname{gph}(\mathscr{A}):=\left\{(x, u) \in \mathbb{R}^{n} \times \mathbb{R}^{n}: u \in \mathscr{A}(x)\right\}$. It is moreover maximal monotone if its graph is not strictly contained in the graph of any other monotone operators.

The best-known example of maximal monotone operator is the subdifferential mapping of functions in $\Gamma_{0}\left(\mathbb{R}^{n}\right)$.

Definition 2.2 ((Averaged) Non-expansive operator) An operator $\mathscr{F}: \mathbb{R}^{n} \rightarrow \mathbb{R}^{n}$ is nonexpansive if

$$
\forall x, y \in \mathbb{R}^{n},\|\mathscr{F}(x)-\mathscr{F}(y)\| \leq\|x-y\| .
$$

For any $\alpha \in] 0,1[, \mathscr{F}$ is called $\alpha$-averaged if there exists a nonexpansive operator $\mathscr{R}$ such that $\mathscr{F}=\alpha \mathscr{R}+(1-\alpha)$ Id.

The class of $\alpha$-averaged operators is closed under relaxation, convex combination and composition [20,21]. In particular when $\alpha=\frac{1}{2}, \mathscr{F}$ is called firmly nonexpansive. Several properties of firmly nonexpansive operators are collected in the following lemma.

Lemma 2.1 Let $\mathscr{F}: \mathbb{R}^{n} \rightarrow \mathbb{R}^{n}$. Then the following statements are equivalent:

(i) $\mathscr{F}$ is firmly nonexpansive;

(ii) Id - F is firmly nonexpansive;

(iii) $2 \mathscr{F}-\mathrm{Id}$ is nonexpansive;

(iv) Given any $\lambda \in] 0,2],(1-\lambda) \operatorname{Id}+\lambda \mathscr{F}$ is $\frac{\lambda}{2}$-averaged;

(v) $\mathscr{F}$ is the resolvent of a maximal monotone operator $A: \mathbb{R}^{n} \rightrightarrows \mathbb{R}^{n}$.

Proof (i) $\Leftrightarrow($ ii) $\Leftrightarrow$ (iii) follow from [20, Proposition 4.2, Corollary 4.29], (i) $\Leftrightarrow($ iv) is [20, Corollary 4.29], and (i) $\Leftrightarrow(v)$ is [20, Corollary 23.8].

Recall the fixed-point operator $\mathscr{F}_{\gamma}$ from (5).

Lemma 2.2 $\mathscr{F}_{\gamma}$ of (5) is firmly nonexpansive.

Proof The reflected resolvents $\operatorname{rprox}_{\gamma J}$ and $\operatorname{rprox}_{\gamma G}$ are nonexpansive [20, Corollary 23.10(ii)], and so is their composition. The claim follows from Lemma 2.1(i) $\Leftrightarrow$ (ii).

Definition 2.3 (Convergent matrices) A matrix $M \in \mathbb{R}^{n \times n}$ is convergent to some $M^{\infty} \mathbb{R}^{n \times n}$ if its power $M^{k}$ is convergent to $M^{\infty} \in \mathbb{R}^{n \times n}$, i.e. if, and only if

$$
\lim _{k \rightarrow \infty}\left\|M^{k}-M^{\infty}\right\|=0 .
$$

The following identity is known as the spectral radius formula

$$
\rho(M)=\lim _{k \rightarrow+\infty}\left\|M^{k}\right\|^{1 / k}
$$


2.2 Angles between Subspaces

In this part we introduce the principal angles and the Friedrichs angle between two subspaces $T_{1}$ and $T_{2}$. Without loss of generality, let $p:=\operatorname{dim}\left(T_{1}\right)$ and $q:=\operatorname{dim}\left(T_{2}\right)$ such that $1 \leq p \leq q \leq n-1$.

Definition 2.4 (Principal angles) The principal angles $\theta_{k} \in\left[0, \frac{\pi}{2}\right], k=1, \ldots, p$ between subspaces $T_{1}$ and $T_{2}$ are defined by, with $u_{0}=v_{0}:=0$ and

$$
\begin{aligned}
\cos \left(\theta_{k}\right):=\left\langle u_{k}, v_{k}\right\rangle=\max \langle u, v\rangle \text { s.t. } & u \in T_{1}, v \in T_{2},\|u\|=1,\|v\|=1, \\
& \left\langle u, u_{i}\right\rangle=\left\langle v, v_{i}\right\rangle=0, i=0, \cdots, k-1 .
\end{aligned}
$$

The principal angles $\theta_{k}$ are unique with $0 \leq \theta_{1} \leq \theta_{2} \leq \cdots \leq \theta_{p} \leq \pi / 2$.

Definition 2.5 (Friedrichs angle) The Friedrichs angle $\left.\left.\theta_{F} \in\right] 0, \frac{\pi}{2}\right]$ between $T_{1}$ and $T_{2}$ is

$$
\begin{aligned}
\cos \left(\theta_{F}\left(T_{1}, T_{2}\right)\right):=\max \langle u, v\rangle \text { s.t. } u & \in T_{1} \cap\left(T_{1} \cap T_{2}\right)^{\perp},\|u\|=1, \\
v & \in T_{2} \cap\left(T_{1} \cap T_{2}\right)^{\perp},\|v\|=1 .
\end{aligned}
$$

The following lemma shows the relation between the Friedrichs and principal angles whose proof can be found in [22, Proposition 3.3].

Lemma 2.3 We have $\theta_{F}\left(T_{1}, T_{2}\right)=\theta_{d+1}>0$ where $d:=\operatorname{dim}\left(T_{1} \cap T_{2}\right)$.

Remark 2.1 One approach to obtain the principal angles is through the singular value decomposition (SVD). For instance, let $X \in \mathbb{R}^{n \times p}$ and $Y \in \mathbb{R}^{n \times q}$ form orthonormal bases for the subspaces $T_{1}$ and $T_{2}$ respectively. Let $U \Sigma V^{T}$ be the SVD of the matrix $X^{T} Y \in \mathbb{R}^{p \times q}$, then $\cos \left(\theta_{k}\right)=\sigma_{k}, k=1,2, \ldots, p$ and $\sigma_{k}$ corresponds to the $k$ 'th largest singular value in $\Sigma$.

\section{Assumptions and Algorithm}

We consider the structured optimization problem

$$
\min _{x \in \mathbb{R}^{n}}[G(x)+J(x)]
$$

where

(A.1) $G, J \in \Gamma_{0}\left(\mathbb{R}^{n}\right)$, the class of proper convex and lsc functions on $\mathbb{R}^{n}$;

(A.2) $\operatorname{ri}(\operatorname{dom}(G)) \cap \operatorname{ri}(\operatorname{dom}(J)) \neq \emptyset$, where $\operatorname{ri}(C)$ is the relative interior of the nonempty convex set $C$, and $\operatorname{dom}(\cdot)$ denotes the domain of the corresponding function;

(A.3) $\operatorname{Argmin}(G+J) \neq \emptyset$, i.e. the set of minimizers is nonempty. 
We also assume that these two functions are simple, meaning that their corresponding proximity operators $\operatorname{prox}_{\gamma J}$ and $\left.\operatorname{prox}_{\gamma G}, \gamma \in\right] 0,+\infty[$, are easy to compute, either exactly or up to a very good approximation. Problem $(\mathcal{P})$ covers a large number of problems in areas such as statistical machine learning, inverse problems, signal and image processing to name a few (see Section 9).

In its exact relaxed form $[8,23,24]$, the iteration of DR reads

$$
\left\{\begin{array}{l}
v_{k+1}=\operatorname{prox}_{\gamma G}\left(2 x_{k}-z_{k}\right), \\
z_{k+1}=\left(1-\lambda_{k}\right) z_{k}+\lambda_{k}\left(z_{k}+v_{k+1}-x_{k}\right), \\
x_{k+1}=\operatorname{prox}_{\gamma J}\left(z_{k+1}\right)
\end{array}\right.
$$

where $\gamma \in] 0,+\infty\left[, \lambda_{k} \in\right] 0,2[$ is the relaxation parameter. The DR scheme (3) can be cast as a fixed-point iteration with respect to $\left\{z_{k}\right\}_{k \in \mathbb{N}}$, i.e.

$$
z_{k+1}=\mathscr{F}_{\gamma, \lambda_{k}}\left(z_{k}\right)
$$

where the fixed-point operator

$$
\mathscr{F}_{\gamma, \lambda}:=(1-\lambda) \operatorname{Id}+\lambda \mathscr{F}_{\gamma} \text { and } \mathscr{F}_{\gamma}:=\frac{\operatorname{rprox}_{\gamma G} \circ \operatorname{rprox}_{\gamma J}+\mathrm{Id}}{2} .
$$

Under assumptions (A.1)-(A.3), and if $\sum_{k \in \mathbb{N}} \lambda_{k}\left(2-\lambda_{k}\right)=+\infty$, it is known that $z_{k}$ converges to some fixed point $z^{\star} \in \operatorname{Fix}\left(\mathscr{F}_{\gamma}\right) \neq \emptyset$, and that the shadow point $x_{k}$ and $v_{k}$ both converge to $x^{\star}:=\operatorname{prox}_{\gamma J}\left(z^{\star}\right) \in \operatorname{Argmin}(G+J)$; see e.g. [20, Corollary 27.7].

In this paper, we consider a non-stationary version of (3), which is described below in Algorithm 1.

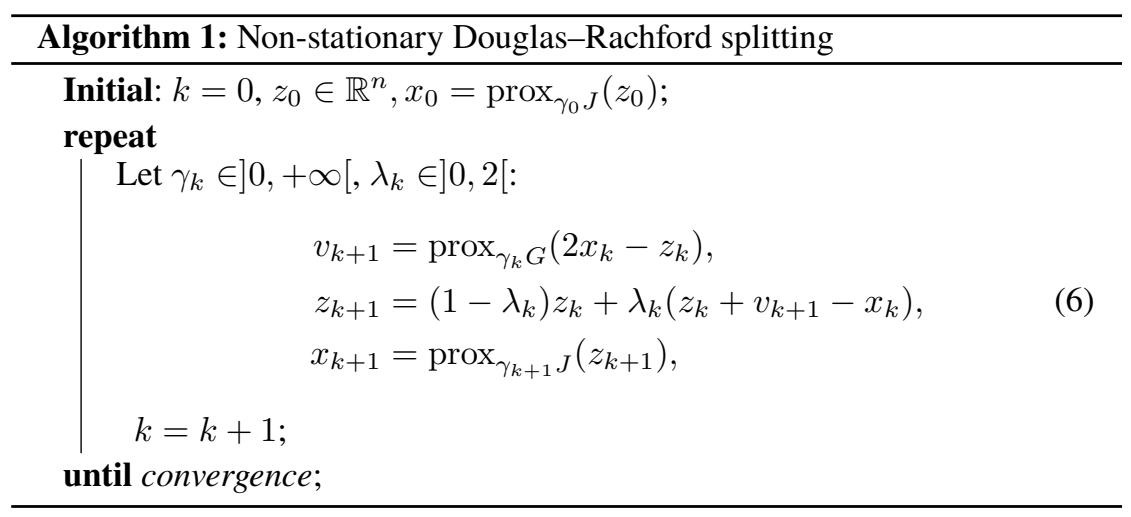

\section{Remark 3.1}

(i) By definition, the DR method is not symmetric with respect to the order of the functions $J$ and $G$, see [25] for a systematic study of the two possible versions in the exact, stationary and unrelaxed case. Nevertheless, all of our statements throughout hold true, with minor adaptations, when the order of $J$ and $G$ is reversed in (6). Note also that the standard DR only accounts for the sum of two functions. Extension to more than two functions is straightforward through a product space trick, see Section 8 for details. 
(ii) This paper consists of two main parts, the first one dealing with global convergence guarantees of (6) (Section 4), and a second one on its the local convergence properties when the involved functions are also partly smooth (Section 6). It is for the sake of the latter that we mainly focus on the finite dimensional setting $\mathbb{R}^{n}$. It is worth pointing out, however, that the global convergence result (Theorem 4.1) also holds for real Hilbert space case where weak convergence can be obtained.

(iii) For global convergence, one can also consider an inexact version of (6) by incorporating additive errors in the computation of $x_{k}$ and $v_{k}$, though we do not elaborate more on this for the sake of local convergence analysis.

\section{Global Convergence}

Recall the operators defined in (5). The nonstationay DR iteration (6) can also be written

$$
z_{k+1}=\mathscr{F}_{\gamma_{k}, \lambda_{k}}\left(z_{k}\right)=\mathscr{F}_{\gamma, \lambda_{k}}\left(z_{k}\right)+\left(\mathscr{F}_{\gamma_{k}, \lambda_{k}}-\mathscr{F}_{\gamma, \lambda_{k}}\right)\left(z_{k}\right)
$$

In plain words, the non-stationary iteration (6) is a perturbed version of the stationary one (3).

Theorem 4.1 (Global convergence) Consider the non-stationary DR iteration (6). Suppose that the following conditions are fulfilled

(H.1) Assumptions (A.1)-(A.3) hold;

(H.2) $\lambda_{k} \in[0,2]$ such that $\sum_{k \in \mathbb{N}} \lambda_{k}\left(2-\lambda_{k}\right)=+\infty$;

(H.3) $\left.\left(\gamma_{k}, \gamma\right) \in\right] 0,+\infty\left[^{2}\right.$ such that $\left\{\lambda_{k}\left|\gamma_{k}-\gamma\right|\right\}_{k \in \mathbb{N}} \in \ell_{+}^{1}$.

Then the sequence $\left\{z_{k}\right\}_{k \in \mathbb{N}}$ converges to a fixed point $z^{\star} \in \operatorname{Fix}\left(\mathscr{F}_{\gamma}\right)$ with $x^{\star}=\operatorname{prox}_{\gamma J}\left(z^{\star}\right) \in \operatorname{Argmin}(G+J)$. Moreover, the shadow sequence $\left\{x_{k}\right\}_{k \in \mathbb{N}}$ and $\left\{v_{k}\right\}_{k \in \mathbb{N}}$ both converge to $x^{\star}$ if $\gamma_{k}$ is convergent.

See Appendix A for the proof.

\section{Remark 4.1}

(i) The conclusions of Theorem 4.1 remain true if $x_{k}$ and $v_{k}$ are computed inexactly with additive errors $\varepsilon_{1, k}$ and $\varepsilon_{2, k}$, provided that $\left\{\lambda_{k}\left\|\varepsilon_{1, k}\right\|\right\}_{k \in \mathbb{N}} \in \ell_{+}^{1}$ and $\left\{\lambda_{k}\left\|\varepsilon_{2, k}\right\|\right\}_{k \in \mathbb{N}} \in \ell_{+}^{1}$.

(ii) The summability assumption (H.3) is weaker than imposing it without $\lambda_{k}$. Indeed, following the discussion in [26, Remark 5.7], take $q \in] 0,1]$, and let $\lambda_{k}=1-\sqrt{1-1 / k}$ and $\left|\gamma_{k}-\gamma\right|=\frac{1+\sqrt{1-1 / k}}{k^{q}}$, then it can be verified that $\left|\gamma_{k}-\gamma\right| \notin \ell_{+}^{1}, \lambda_{k}\left|\gamma_{k}-\gamma\right|=\frac{1}{k^{1+q}} \in \ell_{+}^{1}$ and $\lambda_{k}\left(2-\lambda_{k}\right)=\frac{1}{k} \notin \ell_{+}^{1}$.

(iii) The assumptions made on the sequence $\left\{\gamma_{k}\right\}_{k \in \mathbb{N}}$ imply that $\gamma_{k} \rightarrow \gamma$ (see Lemma A.1). If $\inf _{k \in \mathbb{N}} \lambda_{k}>0$, we have $\left\{\left|\gamma_{k}-\gamma\right|\right\}_{k \in \mathbb{N}} \in \ell_{1}^{+}$, entailing $\gamma_{k} \rightarrow \gamma$, and thus the convergence assumption on $\gamma_{k}$ is superfluous. 


\section{Partial Smoothness}

The concept of partial smoothness was formalized in [9]. This notion, as well as that of identifiable surfaces [27], captures the essential features of the geometry of nonsmoothness which are along the so-called active/identifiable submanifold. For convex functions, a closely related idea is developed in [28]. Loosely speaking, a partly smooth function behaves smoothly as we move along the identifiable submanifold, and sharply if we move transversal to the manifold. In fact, the behaviour of the function and of its minimizers depend essentially on its restriction to this manifold, hence offering a powerful framework for algorithmic and sensitivity analysis theory.

Let $\mathcal{M}$ be a $C^{2}$-smooth embedded submanifold of $\mathbb{R}^{n}$ around a point $x$. To lighten the notations, henceforth we shall state $C^{2}$-manifold instead of $C^{2}$-smooth embedded submanifold of $\mathbb{R}^{n}$. The natural embedding of a submanifold $\mathcal{M}$ into $\mathbb{R}^{n}$ permits to define a Riemannian structure on $\mathcal{M}$, and we simply say $\mathcal{M}$ is a Riemannian manifold. $\mathcal{T}_{\mathcal{M}}(x)$ denotes the tangent space to $\mathcal{M}$ at any point near $x$ in $\mathcal{M}$. More material on manifolds is given in Section C.

We are now in position to formally define the class of partly smooth functions in $\Gamma_{0}\left(\mathbb{R}^{n}\right)$.

Definition 5.1 (Partly smooth function) Let $F \in \Gamma_{0}\left(\mathbb{R}^{n}\right)$, and $x \in \mathbb{R}^{n}$ such that $\partial F(x) \neq \emptyset . F$ is then said to be partly smooth at $x$ relative to a set $\mathcal{M}$ containing $x$ if

(i) Smoothness: $\mathcal{M}$ is a $C^{2}$-manifold around $x, F$ restricted to $\mathcal{M}$ is $C^{2}$ around $x$;

(ii) Sharpness: The tangent space $\mathcal{T}_{\mathcal{M}}(x)$ coincides with $T_{x}=\operatorname{par}(\partial F(x))^{\perp}$;

(iii) Continuity: The set-valued mapping $\partial F$ is continuous at $x$ relative to $\mathcal{M}$. The class of partly smooth functions at $x$ relative to $\mathcal{M}$ is denoted as $\operatorname{PSF}_{x}(\mathcal{M})$.

In fact, local polyhedrality also implies that the subdifferential is locally constant around $x$ along $x+T_{x}$. Capitalizing on the results of [9], it can be shown that under mild transversality assumptions, the set of partly smooth functions is closed under addition and pre-composition by a linear operator. Moreover, absolutely permutationinvariant convex and partly smooth functions of the singular values of a real matrix, i.e. spectral functions, are convex and partly smooth spectral functions of the matrix [29]. Some examples of partly smooth functions will be discussed in Section 9.

The next lemma gives expressions of the Riemannian gradient and Hessian (see Section $\mathrm{C}$ for definitions) of a partly smooth function.

Lemma 5.1 If $F \in \operatorname{PSF}_{x}(\mathcal{M})$, then for any $x^{\prime} \in \mathcal{M}$ near $x$

$$
\nabla_{\mathcal{M}} F\left(x^{\prime}\right)=\mathrm{P}_{T_{x^{\prime}}}\left(\partial F\left(x^{\prime}\right)\right) \text {. }
$$

In turn, for all $h \in T_{x^{\prime}}$

$$
\nabla_{\mathcal{M}}^{2} F\left(x^{\prime}\right) h=\mathrm{P}_{T_{x^{\prime}}} \nabla^{2} \widetilde{F}\left(x^{\prime}\right) h+\mathfrak{W}_{x^{\prime}}\left(h, \mathrm{P}_{T_{x^{\prime}}^{\perp}} \nabla \widetilde{F}\left(x^{\prime}\right)\right),
$$

where $\widetilde{F}$ is any smooth extension (representative) of $F$ on $\mathcal{M}$, and $\mathfrak{W}_{x}(\cdot, \cdot): T_{x} \times T_{x}^{\perp} \rightarrow T_{x}$ is the Weingarten map of $\mathcal{M}$ at $x$.

Proof See [30, Fact 3.3]. 
5.1 Finite Activity Identification

With the above global convergence result at hand, we are now ready to state the finite time activity identification property of the non-stationary DR method.

Let $z^{\star} \in \operatorname{Fix}\left(\mathscr{F}_{\gamma, \lambda}\right)$ and $x^{\star}=\operatorname{prox}_{\gamma J}\left(z^{\star}\right) \in \operatorname{Argmin}(G+J)$, then at convergence of the DR iteration (6), we have the following inclusion holds,

$$
x^{\star}-z^{\star} \in \gamma \partial G\left(x^{\star}\right) \text { and } z^{\star}-x^{\star} \in \gamma \partial J\left(x^{\star}\right) .
$$

Our identification result is built upon this inclusion.

Theorem 5.1 (Finite activity identification) For the DR iteration (6), suppose that (H.1)-(H.3) hold and $\gamma_{k}$ is convergent, entailing that $\left(z_{k}, x_{k}, v_{k}\right) \rightarrow\left(z^{\star}, x^{\star}, x^{\star}\right)$, where $z^{\star} \in \operatorname{Fix}\left(\mathscr{F}_{\gamma, \lambda}\right)$ and $x^{\star} \in \operatorname{Argmin}(G+J)$. Assume that $\inf _{k \in \mathbb{N}} \gamma_{k} \geq \gamma>0$. If $G \in \operatorname{PSF}_{x^{\star}}\left(\mathcal{M}_{x^{\star}}^{G}\right)$ and $J \in \operatorname{PSF}_{x^{\star}}\left(\mathcal{M}_{x^{\star}}^{J}\right)$, and the non-degeneracy condition

$$
x^{\star}-z^{\star} \in \gamma \operatorname{ri}\left(\partial G\left(x^{\star}\right)\right) \text { and } z^{\star}-x^{\star} \in \gamma \operatorname{ri}\left(\partial J\left(x^{\star}\right)\right)
$$

holds. Then

(i) There $\exists K \in \mathbb{N}$ large enough such that for all $k \geq K,\left(v_{k}, x_{k}\right) \in \mathcal{M}_{x^{\star}}^{G} \times \mathcal{M}_{x^{\star}}^{J}$.

(ii) Moreover,

(a) If $\mathcal{M}_{x^{\star}}^{J}=x^{\star}+T_{x^{\star}}^{J}$, then $\forall k \geq K, T_{x^{*}}^{J}=T_{x^{\star}}^{J}$.

(b) If $\mathcal{M}_{x^{\star}}^{G}=x^{\star}+T_{x^{\star}}^{G}$, then $\forall k \geq K, T_{v_{k}}^{G}=T_{x^{\star}}^{G}$.

(c) If $J$ is locally polyhedral around $x^{\star}$, then $x_{k} \in \mathcal{M}_{x^{\star}}^{J}=x^{\star}+T_{x^{\star}}^{J}$ and $T_{x_{k}}^{J}=T_{x^{\star}}^{J}, \forall k \geq K$. Moreover, $\nabla_{\mathcal{M}_{x^{\star}}^{J}} J\left(x_{k}\right)=\nabla_{\mathcal{M}_{x^{\star}}^{J}} J\left(x^{\star}\right)$, and $\nabla_{\mathcal{M}_{x^{\star}}^{J}}^{2} J\left(x_{k}\right)=0$.

(d) If $G$ is locally polyhedral around $x^{\star}$, then $v_{k} \in \mathcal{M}_{x^{\star}}^{G}=x^{\star}+T_{x^{\star}}^{G}$ and $T_{v_{k}}^{G}=T_{x^{\star}}^{G}, \forall k \geq K$. Moreover, $\nabla_{\mathcal{M}_{x^{\star}}^{G}} G\left(v_{k}\right)=\nabla_{\mathcal{M}_{x^{\star}}^{G}} G\left(x^{\star}\right)$, and $\nabla_{\mathcal{M}_{x^{\star}}^{G}}^{2} G\left(v_{k}\right)=0$.

See Appendix B for the proof.

\section{Remark 5.1}

(i) The theorem remains true if the condition on $\gamma_{k}$ is replaced with $\gamma_{k} \geq \underline{\gamma}>0$ and $\lambda_{k} \geq \underline{\lambda}>0$, (use (H.3) in the proof).

(ii) A nondegeneracy condition of the form $v \in \operatorname{ri}(G(x))$ is a geometric generalization of strict complementarity slackness in nonlinear programming. One can easily verify that both conditions coincide when $G=\iota_{C}$, where $C=\{x: F(x) \leq 0\}$, with $F: \mathbb{R}^{n} \rightarrow \mathbb{R}^{p}$, and each component $F_{i}$ is differentiable and convex. Building on the arguments of [31], it is almost a necessary condition for the finite identification of $\mathcal{M}_{x^{\star}}$. Relaxing it in general is a challenging problem.

(iii) In general, we have no identification guarantees for $x_{k}$ and $v_{k}$ if the proximity operators are computed with errors, even if the latter are summable, in which case one can still prove global convergence (see Remark 4.1). The deep reason behind this is that in the exact case, under condition (ND), the proximal mapping of a partly smooth function and that of its restriction to the corresponding active manifold locally agree nearby $x^{\star}$. This property can be easily violated if approximate proximal mappings are involved. 
(iv) When the minimizer is unique, using the fixed-point set characterization of DR, see e.g. [24, Lemma 2.6], it can be shown that condition (ND) is also equivalent to $z^{\star} \in \operatorname{ri}\left(\operatorname{Fix}\left(\mathscr{F}_{\gamma}\right)\right)$.

A bound on the finite identification iteration

In Theorem 5.1, we only mention the existence of $K$, and have not provided an estimate of the number of iterations beyond which finite identification occurs. In fact, there is a situation where the answer is trivial, i.e. $J$ (resp. $G$ ) is the indicator function of a subspace. However, answering such a question in general remains challenging. In the following, we shall give a bound in some important cases.

We start with the following general statement and then show that it holds true typically for indicators of polyhedral sets. Denote $\tau_{k}:=\lambda_{k}\left(2-\lambda_{k}\right)$.

Proposition 5.1 For the DR iteration (6), suppose that (H.1)-(H.3) hold and $\gamma_{k}>0$ is convergent, entailing that $z_{k} \rightarrow z^{\star} \in \operatorname{Fix}\left(\mathscr{F}_{\gamma, \lambda}\right)$ and $\left(v_{k}, x_{k}\right) \rightarrow\left(x^{\star}, x^{\star}\right)$, where $x^{\star} \in \operatorname{Argmin}(G+J)$. Assume that $G \in \operatorname{PSF}_{x^{\star}}\left(\mathcal{M}_{x^{\star}}^{G}\right)$ and $J \in \operatorname{PSF}_{x^{\star}}\left(\mathcal{M}_{x^{\star}}^{J}\right)$, and the non-degeneracy condition (ND) holds. Suppose moreover that $\inf _{k \in \mathbb{N}} \tau_{k} \geq \tau>0$, and the iterates are such that $\partial J\left(x_{k}\right) \subset \operatorname{rbd}\left(\partial J\left(x^{\star}\right)\right)$ whenever $x_{k} \notin \overline{\mathcal{M}}_{x^{\star}}^{\bar{J}}$ and $\partial G\left(v_{k}\right) \subset \operatorname{rbd}\left(\partial G\left(x^{\star}\right)\right)$ whenever $v_{k} \notin \mathcal{M}_{x^{\star}}^{G}$. Then, $\mathcal{M}_{x^{\star}}^{J}$ and $\mathcal{M}_{x^{\star}}^{G}$ will be identified for some $k$ obeying

$$
k \geq \frac{\left\|z_{0}-z^{\star}\right\|^{2}+O\left(\sum_{k \in \mathbb{N}} \lambda_{k}\left|\gamma_{k}-\gamma\right|\right)}{\underline{\gamma}^{2} \underline{\tau} \operatorname{dist}\left(0, \operatorname{rbd}\left(\partial J\left(x^{\star}\right)+\partial G\left(x^{\star}\right)\right)\right)^{2}} .
$$

See Appendix B for the proof.

Observe that the assumption on $\tau_{k}$ automatically implies (H.2). As one intuitively expects, this lower-bound increases as (ND) becomes more demanding.

Example 5.1 (Indicators of polyhedral sets) We will discuss the case of $J$, and the same reasoning applies to $G$. Consider $J$ as the indicator function of a polyhedral set $C^{J}$, i.e.

$$
J(x)=\iota_{C^{J}}(x), \quad \text { where } \quad C^{J}=\left\{x \in \mathbb{R}^{n}:\left\langle c_{i}^{J}, x\right\rangle \leq d_{i}^{J}, i=1, \ldots, m\right\} .
$$

Define $I_{x}^{J}:=\left\{i:\left\langle c_{i}, x\right\rangle=d_{i}\right\}$ the active set at $x$. The normal cone to $C^{J}$ at $x \in C^{J}$ is polyhedral and given by [32, Theorem 6.46]

$$
\left.\partial J(x)=N_{C^{J}}(x)=\operatorname{cone}\left(\left(c_{i}^{J}\right)_{i \in I_{x}^{J}}\right)\right) .
$$

It is immediate then to show that $J$ is partly smooth at $x \in C^{J}$ relative to the affine subspace $\mathcal{M}_{x}^{J}=x+T_{x}^{J}$, where, $T_{x}^{J}=\operatorname{span}\left(\left(c_{i}^{J}\right)_{i \in I_{x}^{J}}\right)^{\perp}$. Let $\mathcal{F}_{x}^{J}$ be the face of $C^{J}$ containing $x$. From [33, Theorem 18.8], one can deduce that

$$
\mathcal{F}_{x}^{J}=\mathcal{M}_{x}^{J} \cap C^{J} .
$$

We then have

$$
\begin{aligned}
& \mathcal{M}_{x^{\star}}^{J} \subsetneq \mathcal{M}_{x}^{J} \underset{(9)}{\Longrightarrow} \mathcal{F}_{x^{\star}}^{J} \subsetneq \mathcal{F}_{x}^{J} \\
& \underset{\text { [34, Proposition 3.4] }}{\Longrightarrow} N_{C^{J}}(x) \text { is a face of (other than) } N_{C^{J}}\left(x^{\star}\right) \\
& \underset{\text { [33, Corollary 18.1.3] }}{\Longrightarrow} \partial J(x) \subset \operatorname{rbd}\left(\partial J\left(x^{\star}\right)\right) .
\end{aligned}
$$


Suppose that $\mathcal{M}_{x^{\star}}^{J}$ has not been identified yet. Therefore, since $x_{k}=\mathrm{P}_{C^{J}}\left(z_{k}\right)=\mathrm{P}_{\mathcal{F}_{x_{k}}^{J} \backslash \mathcal{F}_{x^{\star}}^{J}}\left(z_{k}\right)$, and thanks to (10), this is equivalent to

$$
\text { either } \quad \mathcal{F}_{x^{\star}}^{J} \subsetneq \mathcal{F}_{x_{k}}^{J} \quad \text { or } \quad \mathcal{F}_{x_{k}}^{J} \cap \mathcal{F}_{x^{\star}}^{J}=\emptyset \text {. }
$$

It then follows from (11) and Proposition 5.1 that the number of iterations where $\mathcal{F}_{x^{\star}}^{J} \subsetneq \mathcal{F}_{x_{k}}^{J}$ and $\mathcal{F}_{x^{\star}}^{G} \subsetneq \mathcal{F}_{x_{k}}^{G}$ cannot exceed the bound in (8), and thus identification will happen indeed for some large enough $k$ obeying (8).

\section{Local Linear Convergence}

Building upon the identification results from the previous section, we now turn to the local behaviour of the DR iteration (6) under partial smoothness. The key feature is that, once the active manifolds are identified, the DR iteration locally linearizes (possibly up to first-order). It is then sufficient to control the spectral properties of the matrix appearing in the linearized iteration to exhibit the local linear convergence rate.

\subsection{Locally Linearized Iteration}

Let $z^{\star} \in \operatorname{Fix}\left(\mathscr{F}_{\gamma, \lambda}\right)$ and $x^{\star}=\operatorname{prox}_{\gamma J}\left(z^{\star}\right) \in \operatorname{Argmin}(G+J)$. Define the following two functions

$$
\bar{G}(x):=\gamma G(x)-\left\langle x, x^{\star}-z^{\star}\right\rangle, \bar{J}(x):=\gamma J(x)-\left\langle x, z^{\star}-x^{\star}\right\rangle .
$$

We start with the following key lemma.

Lemma 6.1 Suppose that $G \in \operatorname{PSF}_{x^{\star}}\left(\mathcal{M}_{x^{\star}}^{G}\right)$ and $J \in \operatorname{PSF}_{x^{\star}}\left(\mathcal{M}_{x^{\star}}^{J}\right)$. Define the two matrices

$$
H_{\bar{G}}:=\mathrm{P}_{T_{x^{\star}}^{G}} \nabla_{\mathcal{M}_{x^{\star}}^{G}}^{2} \bar{G}\left(x^{\star}\right) \mathrm{P}_{T_{x^{\star}}^{G}} \text { and } H_{\bar{J}}:=\mathrm{P}_{T_{x^{\star}}^{J}} \nabla_{\mathcal{M}_{x^{\star}}^{J}}^{2} \bar{J}\left(x^{\star}\right) \mathrm{P}_{T_{x^{\star}}^{J}} .
$$

$H_{\bar{G}}$ and $H_{\bar{J}}$ are symmetric positive semi-definite under either of the following cases:

(i) (ND) holds.

(ii) $\mathcal{M}_{x^{\star}}^{G}$ and $\mathcal{M}_{x^{\star}}^{J}$ are affine subspaces.

In turn, the matrices

$$
W_{\bar{G}}:=\left(\mathrm{Id}+H_{\bar{G}}\right)^{-1} \text { and } W_{\bar{J}}:=\left(\operatorname{Id}+H_{\bar{J}}\right)^{-1} \text {, }
$$

are both firmly non-expansive.

Proof Here we prove the case for $J$ since the same arguments apply to $G$ just as well. Claims (i) and (ii) follow from [30, Lemma 4.3] since $J \in \operatorname{PSF}_{x^{\star}}\left(\mathcal{M}_{x^{\star}}^{J}\right)$. Consequently, $W_{\bar{J}}$ is symmetric positive definite with eigenvalues in $\left.] 0,1\right]$. Thus by virtue of [20, Corollary 4.3(ii)], it is firmly non-expansive. 
Now define $M_{\bar{G}}:=\mathrm{P}_{T_{x^{\star}}^{G}} W_{\bar{G}} \mathrm{P}_{T_{x^{\star}}^{G}}$ and $M_{\bar{J}}:=\mathrm{P}_{T_{x^{\star}}^{J}} W_{\bar{J}} \mathrm{P}_{T_{x^{\star}}^{J}}$, and the matrices

$$
\begin{aligned}
M & :=\mathrm{Id}+2 M_{\bar{G}} M_{\bar{J}}-M_{\bar{G}}-M_{\bar{J}}=\frac{1}{2} \mathrm{Id}+\frac{1}{2}\left(2 M_{\bar{G}}-\mathrm{Id}\right)\left(2 M_{\bar{J}}-\mathrm{Id}\right), \\
M_{\lambda} & :=(1-\lambda) \mathrm{Id}+\lambda M, \quad \lambda \in] 0,2[.
\end{aligned}
$$

We have the following locally linearized version of (6).

Proposition 6.1 (Locally linearized DR iteration) For the DR iteration (6), suppose that (H.1)-(H.3) hold and $\gamma_{k}>0$ is convergent, entailing that $z_{k} \rightarrow z^{\star} \in \operatorname{Fix}\left(\mathscr{F}_{\gamma, \lambda}\right)$ and $v_{k}, x_{k} \rightarrow x^{\star} \in \operatorname{Argmin}(G+J)$. Assume also that $G \in \operatorname{PSF}_{x^{\star}}\left(\mathcal{M}_{x^{\star}}^{G}\right)$ and $J \in \operatorname{PSF}_{x^{\star}}\left(\mathcal{M}_{x^{\star}}^{J}\right)$, and the non-degeneracy condition (ND) holds. Assume also that $\left.\lambda_{k} \rightarrow \lambda \in\right] 0,2\left[\right.$. Then $M$ is firmly non-expansive and $M_{\lambda}$ is $\frac{\lambda}{2}$-averaged. Moreover, for all $k$ large enough, we have

$$
z_{k+1}-z^{\star}=M_{\lambda}\left(z_{k}-z^{\star}\right)+\psi_{k}+\phi_{k}
$$

where $\left\|\psi_{k}\right\|=o\left(\left\|z_{k}-z^{\star}\right\|\right)$ and $\phi_{k}=O\left(\lambda_{k}\left|\gamma_{k}-\gamma\right|\right)$. $\psi_{k}$ and $\phi_{k}$ vanish when $G$ and $J$ are locally polyhedral around $x^{\star}$ and $\left(\gamma_{k}, \lambda_{k}\right)$ are chosen constant in $] 0,+\infty[\times] 0,2[$.

See Appendix C for the proof.

Remark 6.1 If $\phi_{k}=o\left(\left\|z_{k}-z^{\star}\right\|\right)$, then the rest in (17) is $o\left(z_{k}-z^{\star}\right)$. However, this is of little practical interest as $z^{\star}$ is unknown.

Next we derive a characterization of the spectral properties of $M_{\lambda}$, which in turn, will allow to study the linear convergence rates of its powers $M_{\lambda}^{k}$ to the limit $M^{\infty}$. Recall the notion of convergent matrices from Definition 2.3. To lighten the notation, we will set $S_{x^{\star}}^{J}:=\left(T_{x^{\star}}^{J}\right)^{\perp}$ and $S_{x^{\star}}^{G}:=\left(T_{x^{\star}}^{G}\right)^{\perp}$.

Lemma 6.2 Suppose that $\lambda \in] 0,2[$, then,

(i) $M_{\lambda}$ is convergent with

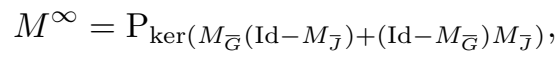

and we have

$$
\forall k \in \mathbb{N}, M_{\lambda}^{k}-M^{\infty}=\left(M_{\lambda}-M^{\infty}\right)^{k} \text { and } \rho\left(M_{\lambda}-M^{\infty}\right)<1 .
$$

In particular, if

$T_{x^{\star}}^{J} \cap T_{x^{\star}}^{G}=\{0\}, \quad \operatorname{span}\left(\operatorname{Id}-M_{\bar{J}}\right) \cap S_{x^{\star}}^{G}=\{0\}$ and $\operatorname{span}\left(\mathrm{Id}-M_{\bar{G}}\right) \cap T_{x^{\star}}^{G}=\{0\}$, then $M^{\infty}=0$.

(ii) Given any $\rho \in] \rho\left(M_{\lambda}-M^{\infty}\right), 1[$, there is $K$ large enough such that for all $k \geq K$,

$$
\left\|M_{\lambda}^{k}-M^{\infty}\right\|=O\left(\rho^{k}\right)
$$


(iii) If, moreover, $G$ and $J$ are locally polyhedral around $x^{\star}$, then $M_{\lambda}$ is normal (i.e. $\left.M_{\lambda}^{T} M_{\lambda}=M_{\lambda} M_{\lambda}^{T}\right)$ and converges linearly to $\mathrm{P}_{\left(T_{x^{\star}}^{J} \cap T_{x^{\star}}^{G}\right) \oplus\left(S_{x^{\star}}^{J} \cap S_{x^{\star}}^{G}\right)}$ with the optimal rate

$$
\sqrt{(1-\lambda)^{2}+\lambda(2-\lambda) \cos ^{2}\left(\theta_{F}\left(T_{x^{\star}}^{J}, T_{x^{\star}}^{G}\right)\right)}<1 .
$$

In particular, if $T_{x^{\star}}^{J} \cap T_{x^{\star}}^{G}=S_{x^{\star}}^{J} \cap S_{x^{\star}}^{G}=\{0\}$, then $M_{\lambda}$ converges linearly to 0 with the optimal rate

$$
\sqrt{(1-\lambda)^{2}+\lambda(2-\lambda) \cos ^{2}\left(\theta_{1}\left(T_{x^{\star}}^{J}, T_{x^{\star}}^{G}\right)\right)}<1 .
$$

See Appendix C for the proof.

Combining Proposition 6.1 and Lemma 6.2, we have the following equivalent characterization of the locally linearized iteration.

Corollary 6.1 For the linearized iteration in Proposition 6.1, the following holds.

(i) (17) is equivalent to

$\left(\mathrm{Id}-M^{\infty}\right)\left(z_{k+1}-z^{\star}\right)=\left(M_{\lambda}-M^{\infty}\right)\left(\mathrm{Id}-M^{\infty}\right)\left(z_{k}-z^{\star}\right)+\left(\mathrm{Id}-M^{\infty}\right) \psi_{k}+\phi_{k}$.

(ii) If $G$ and $J$ are locally polyhedral around $x^{\star}$ and $\left(\gamma_{k}, \lambda_{k}\right)$ are constant in ] $0,+\infty[\times] 0,2[$, then

$$
z_{k+1}-z^{\star}=\left(M_{\lambda}-M^{\infty}\right)\left(z_{k}-z^{\star}\right) .
$$

The direction $\Rightarrow$ is easy, the converse needs more arguments. See Appendix C for the proof.

\subsection{Local Linear Convergence}

We are now in position to present the local linear convergence of the DR iteration (6).

Theorem 6.1 (Local linear convergence of DR) For the DR iteration (6), suppose that Proposition 6.1 holds. Recall $M^{\infty}$ from Lemma 6.2. The following holds:

(i) Given any $\rho \in] \rho\left(M_{\lambda}-M^{\infty}\right), 1[$, there exists $K \in \mathbb{N}$ large enough such that for all $k \geq K$, if $\lambda_{k}\left|\gamma_{k}-\gamma\right|=O\left(\eta^{k}\right)$ for $0 \leq \eta<\rho$, then

$$
\left\|\left(\operatorname{Id}-M^{\infty}\right)\left(z_{k}-z^{\star}\right)\right\|=O\left(\rho^{k-K}\right) .
$$

(ii) If $G$ and $J$ are locally polyhedral around $x^{\star}$ and $\left(\gamma_{k}, \lambda_{k}\right) \equiv(\gamma, \lambda)$, where $(\gamma, \lambda) \in] 0,+\infty[\times] 0,2[$, then there exists $K \in \mathbb{N}$ large enough such that for all $k \geq K$,

$$
\left\|z_{k}-z^{\star}\right\| \leq \rho^{k-K}\left\|z_{K}-z^{\star}\right\|
$$

where the convergence rate $\rho=\sqrt{(1-\lambda)^{2}+\lambda(2-\lambda) \cos ^{2}\left(\theta_{F}\left(T_{x^{\star}}^{J}, T_{x^{\star}}^{G}\right)\right)}$, in $[0,1[$, is optimal. 
See Appendix C for the proof.

\section{Remark 6.2}

(i) If $M^{\infty}=0$ in (i) or in the situation of (ii), we also have local linear convergence of $x_{k}$ and $v_{k}$ to $x^{\star}$ by non-expansiveness of the proximity operator.

(ii) The condition on $\phi_{k}$ in Theorem 6.1(i) amounts to saying that $\gamma_{k}$ should converge fast enough to $\gamma$. Otherwise, the local convergence rate could be dominated by that of $\phi_{k}$. In particular, if $\phi_{k}$ converges sub-linearly to 0 , then the local convergence rate will eventually become sublinear. See Figure 5 in the numerical experiments section.

(iii) For Theorem 6.1(ii), it can be observed that the best rate is obtained for $\lambda=1$. This has been also pointed out in [10] for basis pursuit. This assertion is however only valid for the local convergence behaviour and does not mean in general that the DR will be globally faster for $\lambda_{k} \equiv 1$.

(iv) Observe also that the local linear convergence rate does not depend on $\gamma$ when both $G$ and $J$ are locally polyhedral around $x^{\star}$. This means that the choice of $\gamma_{k}$ only affects the number of iterations needed for finite identification. For general partly smooth functions, $\gamma_{k}$ influences both the identification time and the local linear convergence rate, since $M_{\lambda}$ depends on it through the matrices $W_{\bar{G}}$ and $W_{\bar{J}}(\gamma$ weights the Riemannian Hessians of $\bar{G}$ and $\bar{J}$; see (13)-(15).

\section{Finite Convergence}

We are now ready to characterize situations where finite convergence of DR occurs.

Theorem 7.1 Assume that the unrelaxed stationary DR iteration is used (i.e., $\left.\gamma_{k} \equiv \gamma \in\right] 0,+\infty\left[\right.$ and $\left.\lambda_{k} \equiv 1\right)$, such that $\left(z_{k}, x_{k}, v_{k}\right) \rightarrow\left(z^{\star}, x^{\star}, x^{\star}\right)$, where G and $J$ are locally polyhedral nearby $x^{\star}$. Suppose that either $J$ or $G$ is locally $C^{2}$ at $x^{\star}$. Then the DR sequences $\left\{z_{k}, x_{k}, v_{k}\right\}_{k \in \mathbb{N}}$ converge in finitely many steps to $\left(z^{\star}, x^{\star}, x^{\star}\right)$.

Proof We will prove the statement when $J$ is locally $C^{2}$ at $x^{\star}$, and the same reasoning holds if the assumption is on $G$. Local $C^{2}$-smoothness of $J$ at $x^{\star}$ entails that $\partial J\left(x^{\star}\right)=\left\{\nabla J\left(x^{\star}\right)\right\}$ and $J$ is partly smooth at $x^{\star}$ relative to $\mathcal{M}_{x^{\star}}^{J}=\mathbb{R}^{n}$. Moreover, the non-degeneracy condition (ND) is in force. It then follows from Proposition 6.1 and Lemma 6.2(i) that there exists $K \in \mathbb{N}$ large enough such that

$$
\forall k \geq K, \quad z_{k+1}-z^{\star}=\mathrm{P}_{T_{x^{\star}}^{G}}\left(z_{k}-z^{\star}\right) \Rightarrow \forall k \geq K+1, \quad z_{k}-z^{\star} \in T_{x^{\star}}^{G},
$$

whence we conclude that

$$
\forall k \geq K+1, \quad z_{k}=z_{k+1}=\cdots=z^{\star} .
$$

DR is known (see, e.g., [8, Theorem 6]) to be a special case of the exact proximal point algorithm (PPA) with constant step-size $\gamma_{k} \equiv 1$. This suggests that many results related to PPA can be carried over to DR. For instance, finite convergence of PPA has been studied in [35,36] under different conditions. However, [8, Theorem 9] 
gave a negative result that suggests that these previous conditions sufficient for finite termination of PPA can be difficult or impossible to carry over to DR even for the polyhedral case. The authors in [19] considered the unrelaxed and stationary DR for solving the convex feasibility problem

$$
\text { Find a point in } C_{1} \cap C_{2} \text {, }
$$

where $C_{1}$ and $C_{2}$ are nonempty closed convex sets in $\mathbb{R}^{n}, C_{1} \cap C_{2} \neq \emptyset, C_{1}$ is an affine subspace and $C_{2}$ is a polyhedron. They established finite convergence under Slater's condition

$$
C_{1} \cap \operatorname{int}\left(C_{2}\right) \neq \emptyset .
$$

They also provided examples where this condition holds where the conditions of [35, 36] for finite convergence do not apply.

Specializing our result to $G=\iota_{C_{1}}$ and $J=\iota_{C_{2}}$, then under Slater's condition, if $x^{\star} \in C_{1} \cap \operatorname{int}\left(C_{2}\right)$, we have $G$ is partly smooth at any $x \in C_{1}$ relative to $C_{1}$ with $T_{x^{\star}}^{G}=\operatorname{par}\left(C_{1}\right)$ (i.e. a translate of $C_{1}$ to the origin), and $\partial J\left(x^{\star}\right)=N_{C_{2}}\left(x^{\star}\right)=\{0\}$, and we recover the result of [19]. In fact, [19, Theorem 3.7] shows that the cluster point $x^{\star}$ is always an interior point regardless of the starting point of DR. The careful reader may have noticed that in the current setting, thanks to Example 5.1, the estimate in (5.1) gives a bound on the finite convergence iteration.

\section{More than Two Functions}

We now want to tackle the problem of solving

$$
\min _{x \in \mathbb{R}^{n}} \sum_{i=1}^{m} J_{i}(x),
$$

where

(A'.1) $J_{i} \in \Gamma_{0}\left(\mathbb{R}^{n}\right), \forall i=1, \cdots, m$;

(A'.2) $\bigcap_{1 \leq i \leq m} \operatorname{ri}\left(\operatorname{dom}\left(J_{i}\right)\right) \neq \emptyset$;

(A'.3) $\operatorname{Argmin}\left(\sum_{i=1}^{m} J_{i}\right) \neq \emptyset$.

In fact, problem $\left(\mathcal{P}_{m}\right)$ can be equivalently reformulated as $(\mathcal{P})$ in a product space, see e.g. $[37,38]$. Let $\mathcal{H}=\underbrace{\mathbb{R}^{n} \times \cdots \times \mathbb{R}^{n}}_{m \text { times }}$ endowed with the scalar inner-product and norm

$$
\forall \boldsymbol{x}, \boldsymbol{y} \in \mathcal{H},\langle\boldsymbol{x}, \boldsymbol{y}\rangle=\sum_{i=1}^{m}\left\langle x_{i}, y_{i}\right\rangle,\|\boldsymbol{x}\|=\sqrt{\sum_{i=1}^{m}\left\|x_{i}\right\|^{2}} .
$$

Let $\mathcal{S}=\left\{\boldsymbol{x}=\left(x_{i}\right)_{i} \in \mathcal{H}: x_{1}=\cdots=x_{m}\right\}$ and its orthogonal complement $\mathcal{S}^{\perp}=\left\{\boldsymbol{x}=\left(x_{i}\right)_{i} \in \mathcal{H}: \sum_{i=1}^{m} x_{i}=0\right\}$. Define the canonical isometry $\boldsymbol{C}: \mathbb{R}^{n} \rightarrow \mathcal{S}, x \mapsto(x, \cdots, x)$, we have $\mathrm{P}_{\mathcal{S}}(\boldsymbol{z})=\boldsymbol{C}\left(\frac{1}{m} \sum_{i=1}^{m} z_{i}\right)$.

Problem $\left(\mathcal{P}_{m}\right)$ is now equivalent to

$$
\min _{\boldsymbol{x} \in \mathcal{H}} \boldsymbol{J}(\boldsymbol{x})+\boldsymbol{G}(\boldsymbol{x}), \text { where } \boldsymbol{J}(\boldsymbol{x})=\sum_{i=1}^{m} J_{i}\left(x_{i}\right) \text { and } \boldsymbol{G}(\boldsymbol{x})=\iota \mathcal{S}(\boldsymbol{x}),
$$

which has the same structure on $\mathcal{H}$ as $(\mathcal{P})$ on $\mathbb{R}^{n}$. 
Obviously, $\boldsymbol{J}$ is separable and therefore,

$$
\operatorname{prox}_{\gamma \boldsymbol{J}}(\boldsymbol{x})=\left(\operatorname{prox}_{\gamma J_{i}}\left(x_{i}\right)\right)_{i} .
$$

Let $\boldsymbol{x}^{\star}=\boldsymbol{C}\left(x^{\star}\right)$. Clearly, $\boldsymbol{G}$ is polyhedral, hence partly smooth relative to $\mathcal{S}$ with $\boldsymbol{T}_{\boldsymbol{x}^{\star}}^{\boldsymbol{G}}=\mathcal{S}$. Suppose that $J_{i} \in \mathrm{PSF}_{x^{\star}}\left(\mathcal{M}_{x^{\star}}^{J_{i}}\right)$ for each $i$. Denote $\boldsymbol{T}_{\boldsymbol{x}^{\star}}^{\boldsymbol{J}}=\chi_{i} T_{x^{\star}}^{J_{i}}$ and $\boldsymbol{S}_{\boldsymbol{x}^{\star}}^{\boldsymbol{J}}=\chi_{i}\left(T_{x^{\star}}^{J_{i}}\right)^{\perp}$. Similarly to (14), define

$$
\boldsymbol{H}_{\overline{\boldsymbol{J}}}:=\mathrm{P}_{\boldsymbol{T}_{\boldsymbol{x}^{\star}}^{J}} \nabla^{2} \overline{\boldsymbol{J}}\left(x^{\star}\right) \mathrm{P}_{\boldsymbol{T}_{\boldsymbol{x}^{\star}}^{J}} \text { and } \boldsymbol{W}_{\overline{\boldsymbol{J}}}:=\left(\mathbf{I d}+\boldsymbol{H}_{\overline{\boldsymbol{J}}}\right)^{-1},
$$

where $\overline{\boldsymbol{J}}(\boldsymbol{x}):=\gamma \sum_{i=1}^{m} \widetilde{J}_{i}\left(x_{i}\right)-\left\langle\boldsymbol{x}, \boldsymbol{z}^{\star}-\boldsymbol{x}^{\star}\right\rangle, \widetilde{J}_{i}$ is the smooth representation of $J_{i}$ on $\mathcal{M}_{x^{\star}}^{J_{i}}$, and Id is the identity operatror on $\mathcal{H}$. Since $\boldsymbol{G}$ is polyhedral, we have $\boldsymbol{W}_{\bar{G}}=\mathbf{I d}$. Now we can provide the product space form of (16), which reads

$$
\begin{aligned}
& \boldsymbol{M}=\mathbf{I d}+2 \mathrm{P}_{\boldsymbol{T}_{\boldsymbol{x}^{\star}}^{G}} \mathrm{P}_{\boldsymbol{T}_{\boldsymbol{x}^{\star}}^{J}} \boldsymbol{W}_{\overline{\boldsymbol{J}}} \mathrm{P}_{\boldsymbol{T}_{\boldsymbol{x}^{\star}}^{J}}-\mathrm{P}_{\boldsymbol{T}_{\boldsymbol{x}^{\star}}^{G}}-\mathrm{P}_{\boldsymbol{T}_{\boldsymbol{x}^{\star}}^{J}} \boldsymbol{W}_{\overline{\boldsymbol{J}}} \mathrm{P}_{\boldsymbol{T}_{\boldsymbol{x}^{\star}}^{J}} \\
& =\frac{1}{2} \mathbf{I d}+\mathrm{P}_{\boldsymbol{T}_{\boldsymbol{x}^{\star}}^{G}}\left(2 \mathrm{P}_{\boldsymbol{T}_{\boldsymbol{x}^{\star}}^{J}} \boldsymbol{W}_{\overline{\boldsymbol{J}}} \mathrm{P}_{\boldsymbol{T}_{\boldsymbol{x}^{\star}}^{J}}-\mathbf{I d}\right)-\frac{1}{2}\left(2 \mathrm{P}_{\boldsymbol{T}_{\boldsymbol{x}^{\star}}^{J}} \boldsymbol{W}_{\overline{\boldsymbol{J}}} \mathrm{P}_{\boldsymbol{T}_{\boldsymbol{x}^{\star}}^{J}}-\mathbf{I d}\right) \\
& =\frac{1}{2} \mathbf{I d}+\frac{1}{2}\left(2 \mathrm{P}_{\boldsymbol{T}_{\boldsymbol{x}^{\star}}^{\boldsymbol{G}}}-\mathbf{I d}\right)\left(2 \mathrm{P}_{\boldsymbol{T}_{\boldsymbol{x}^{\star}}^{J}} \boldsymbol{W}_{\overline{\boldsymbol{J}}} \mathrm{P}_{\boldsymbol{T}_{\boldsymbol{x}^{\star}}^{J}}-\mathbf{I d}\right),
\end{aligned}
$$

and $\boldsymbol{M}_{\lambda}:=(1-\lambda) \mathbf{I d}+\lambda \boldsymbol{M}$. Owing to Lemma 6.2, we have

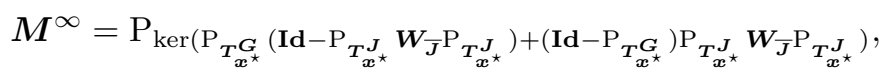

and when all $J_{i}$ 's are locally polyhedral nearby $x^{\star}, M^{\infty}$ specializes to

$$
M^{\infty}=\mathrm{P}_{\left(\boldsymbol{T}_{x^{\star}}^{J} \cap \mathcal{S}\right) \oplus\left(\boldsymbol{S}_{x^{\star}}^{J} \cap \mathcal{S}^{\perp}\right)} .
$$

Corollary 8.1 Suppose that (A'.1)-(A'.3) and (H.2)-(H.3) holds. Consider the sequence $\left\{\boldsymbol{z}_{k}, \boldsymbol{x}_{k}, \boldsymbol{v}_{k}\right\}_{k \in \mathbb{N}}$ provided by the non-stationary DR method (6) applied to solve $(\mathcal{P})$. Then,

(i) $\left(\boldsymbol{z}_{k}, \boldsymbol{x}_{k}, \boldsymbol{v}_{k}\right)$ converges to $\left(\boldsymbol{z}^{\star}, \boldsymbol{x}^{\star}, \boldsymbol{x}^{\star}\right)$, where $\boldsymbol{x}^{\star}=\boldsymbol{C}\left(x^{\star}\right)$ and $x^{\star}$ is a global minimizer of $\left(\mathcal{P}_{m}\right)$.

(ii) Assume, moreover, that $\gamma_{k} \geq \underline{\gamma}>0$ and $\gamma_{k} \rightarrow \gamma, J_{i} \in \operatorname{PSF}_{x^{\star}}\left(\mathcal{M}_{x^{\star}}^{J_{i}}\right)$ and

$$
\boldsymbol{z}^{\star} \in \boldsymbol{x}^{\star}+\gamma \mathrm{ri}\left(\chi_{i} \partial J_{i}\left(x^{\star}\right)\right) \cap \mathcal{S}^{\perp} .
$$

(ND)

Then,

(a) for all $k$ large enough, $\boldsymbol{x}_{k} \in \mathrm{X}_{i} \mathcal{M}_{x^{\star}}^{J_{i}}$.

(b) in addition, if $\left.\lambda_{k} \rightarrow \lambda \in\right] 0,2[$, then given any $\rho \in] \rho\left(\boldsymbol{M}_{\lambda}-\boldsymbol{M}^{\infty}\right), 1$, there exists $K \in \mathbb{N}$ large enough such that for all $k \geq K$, if $\lambda_{k}\left|\gamma_{k}-\gamma\right|=O\left(\eta^{k}\right)$ where $0 \leq \eta<\rho$, then

$$
\left\|\left(\mathbf{I} \mathbf{d}-\boldsymbol{M}^{\infty}\right)\left(\boldsymbol{z}_{k}-\boldsymbol{z}^{\star}\right)\right\|=O\left(\rho^{k-K}\right) .
$$

In particular, if all $J_{i}$ 's are locally polyhedral around $x^{\star}$ and $\left.\left(\gamma_{k}, \lambda_{k}\right) \equiv(\gamma, \lambda) \in\right] 0,+\infty[\times] 0,2\left[\right.$, then $\boldsymbol{z}_{k}\left(\right.$ resp. $\left.x_{k}:=\frac{1}{m} \sum_{i=1}^{m} \boldsymbol{x}_{k, i}\right)$ converges locally linearly to $z^{\star}\left(\right.$ resp. $\left.x^{\star}\right)$ at the optimal rate

$$
\rho=\sqrt{(1-\lambda)^{2}+\lambda(2-\lambda) \cos ^{2}\left(\theta_{F}\left(\boldsymbol{T}_{x^{\star}}^{\boldsymbol{J}}, \mathcal{S}\right)\right)} \in[0,1[.
$$


Proof

(i) Apply Theorem 4.1 to $(\mathcal{P})$.

(ii) (a) By the separability rule, we have $\boldsymbol{J} \in \mathrm{PSF}_{\boldsymbol{x}^{\star}}\left(X_{i} \mathcal{M}_{x^{\star}}^{J_{i}}\right)$, see [9, Proposition 4.5]. We have also $\partial \boldsymbol{G}\left(\boldsymbol{x}^{\star}\right)=N_{\mathcal{S}}\left(\boldsymbol{x}^{\star}\right)=\mathcal{S}^{\perp}$. Then (ND) is simply a specialization of condition (ND) to $\operatorname{problem}(\mathcal{P})$. The claim then follows from Theorem 5.1.

(b) This is a direct consequence of Theorem 6.1. For the local linear convergence of $x_{k}$ to $x^{\star}$ in the last part, observe that

$$
\begin{aligned}
\left\|x_{k}-x^{\star}\right\|^{2} & =\left\|\frac{1}{m} \sum_{i=1}^{m} \boldsymbol{x}_{k, i}-\frac{1}{m} \sum_{i=1}^{m} \boldsymbol{x}_{i}^{\star}\right\|^{2} \leq \frac{1}{m} \sum_{i=1}^{m}\left\|\boldsymbol{x}_{k, i}-\boldsymbol{x}_{i}^{\star}\right\|^{2} \\
& =\frac{1}{m} \sum_{i=1}^{m}\left\|\operatorname{prox}_{\gamma J_{i}}\left(\boldsymbol{z}_{k, i}\right)-\operatorname{prox}_{\gamma J_{i}}\left(\boldsymbol{z}_{i}^{\star}\right)\right\|^{2} \\
& \leq \frac{1}{m} \sum_{i=1}^{m}\left\|\boldsymbol{z}_{k, i}-\boldsymbol{z}_{i}^{\star}\right\|^{2}=\frac{1}{m}\left\|\boldsymbol{z}_{k}-\boldsymbol{z}^{\star}\right\|^{2} .
\end{aligned}
$$

We also have the following corollary of Theorem 7.1.

Corollary 8.2 Assume that the unrelaxed stationary DR iteration is used (i.e., $\left.\gamma_{k} \equiv \gamma \in\right] 0,+\infty\left[\right.$ and $\left.\lambda_{k} \equiv 1\right)$, such that $\left(\boldsymbol{z}_{k}, \boldsymbol{x}_{k}, \boldsymbol{v}_{k}\right) \rightarrow\left(\boldsymbol{z}^{\star}, \boldsymbol{C}\left(x^{\star}\right), \boldsymbol{C}\left(x^{\star}\right)\right)$, where, $\forall i, J_{i}$ is locally polyhedral nearby $x^{\star}$ and is differentiable at $x^{\star}$. Then the sequences $\left\{\boldsymbol{z}_{k}, \boldsymbol{x}_{k}, \boldsymbol{v}_{k}, \frac{1}{m} \sum_{i=1}^{m} \boldsymbol{x}_{k, i}\right\}_{k \in \mathbb{N}}$ converge in finitely many steps to $\left(\boldsymbol{z}^{\star}, \boldsymbol{C}\left(x^{\star}\right), \boldsymbol{C}\left(x^{\star}\right), x^{\star}\right)$.

\section{Numerical Experiments}

\subsection{Examples of Tested Partly Smooth Functions}

Table 1 provides some examples of partly smooth functions that we will use throughout this section in our numerical experiments. These functions are widely used in the literature to regularize a variety of problems in signal/image processing, machine learning and statistics, see e.g. [39] and references therein for details. The corresponding Riemannian gradients can also be found in [39]. Since the $\ell_{1}, \ell_{\infty}$ and the (anisotropic) total variation semi-norm are polyhedral, their Riemannian Hessian vanishes. The Riemannian Hessians for the $\ell_{1,2}$ nand the nuclear norm are also provided in [39].

Affinely-constrained minimization

Let us first consider the affine-constrained minimization problem

$$
\min _{x \in \mathbb{R}^{n}} J(x) \quad \text { subject to } L x=L x_{\mathrm{ob}},
$$

where $L: \mathbb{R}^{n} \rightarrow \mathbb{R}^{m}$ is a linear operator, $x_{\mathrm{ob}} \in \mathbb{R}^{n}$ is known and $J \in \Gamma_{0}\left(\mathbb{R}^{n}\right)$. Problem (22) is of importance in various areas to find regularized solutions to linear equations (one can think for instance of the active area of compressed sensing, matrix completion, and so on). By identifying $G$ with the indicator function of the affine 
Table 1 Examples of partly smooth functions. $D_{\text {DIF }}$ stands for the finite differences operator.

\begin{tabular}{|c|c|c|}
\hline Function & Expression & Partial smooth manifold \\
\hline$\ell_{1}$-norm & $\|x\|_{1}=\sum_{i=1}^{n}\left|x_{i}\right|$ & $\begin{array}{l}\mathcal{M}=T_{x}=\left\{z \in \mathbb{R}^{n}: I_{z} \subseteq I_{x}\right\} \\
I_{x}=\left\{i: x_{i} \neq 0\right\}\end{array}$ \\
\hline$\ell_{1,2}$-norm & $\sum_{i=1}^{m}\left\|x_{b_{i}}\right\|$ & $\begin{array}{l}\mathcal{M}=T_{x}=\left\{z \in \mathbb{R}^{n}: I_{z} \subseteq I_{x}\right\} \\
I_{x}=\left\{i: x_{b_{i}} \neq 0\right\}\end{array}$ \\
\hline$\ell_{\infty}$-norm & $\max _{i=1, \ldots, n}\left|x_{i}\right|$ & $\begin{array}{l}\mathcal{M}=T_{x}=\left\{z \in \mathbb{R}^{n}: z_{I_{x}} \in \mathbb{R}, \operatorname{sign}\left(x_{I_{x}}\right)\right\} \\
I_{x}=\left\{i:\left|x_{i}\right|=\|x\|_{\infty}\right\}\end{array}$ \\
\hline TV semi-norm & $\|x\|_{\mathrm{TV}}=\left\|D_{\mathrm{DIF}} x\right\|_{1}$ & $\begin{array}{l}\mathcal{M}=T_{x}=\left\{z \in \mathbb{R}^{n}: I_{D_{\mathrm{DIF}} z} \subseteq I_{D_{\mathrm{DIF}} x}\right\} \\
I_{D_{\mathrm{DIF}} x}=\left\{i:\left(D_{\mathrm{DIF}} x\right)_{i} \neq 0\right\}\end{array}$ \\
\hline Nuclear norm & $\|x\|_{*}=\sum_{i=1}^{r} \sigma(x)$ & $\begin{array}{l}\mathcal{M}=\left\{z \in \mathbb{R}^{n_{1} \times n_{2}}: \operatorname{rank}(z)=\operatorname{rank}(x)=r\right\} \\
\sigma(x) \text { singular values of } x\end{array}$ \\
\hline
\end{tabular}

constraint $C:=\left\{x \in \mathbb{R}^{n}: L x_{\mathrm{ob}}=L x\right\}=x_{\mathrm{ob}}+\operatorname{ker}(L)$, it is immediate to see that $G$ is indeed polyhedral and partly smooth at any $x \in C$ relative to $C$.

We here solve (22) with $J$ being the $\ell_{1}, \ell_{\infty}, \ell_{1,2}$ and nuclear norms. For all these cases, the proximity operator of $J$ can be computed very easily. In all these experiments, $L$ is drawn randomly from the standard Gaussian ensemble, i.e. compressed sensing/matrix completion scenario, with the following settings:

$$
\begin{array}{cl}
\ell_{1} \text {-norm } & (m, n)=(48,128), x_{\mathrm{ob}} \text { is sparse with } 8 \text { nonzero entries; } \\
\ell_{1,2} \text {-norm } & (m, n)=(48,128), x_{\mathrm{ob}} \text { has } 3 \text { nonzero blocks of size } 4 ; \\
\ell_{\infty} \text {-norm } & (m, n)=(123,128), x_{\mathrm{ob}} \text { has } 10 \text { saturating components; } \\
\text { Nuclear norm } & (m, n)=(500,1024), x_{\mathrm{ob}} \in \mathbb{R}^{32 \times 32} \text { and } \operatorname{rank}\left(x_{\mathrm{ob}}\right)=4 .
\end{array}
$$

For each setting, the number of measurements is sufficiently large so that one can prove that the minimizer $x^{\star}$ is unique, and in particular that $\operatorname{ker}(L) \cap T_{x^{\star}}^{J}=\{0\}$ (with high probability); see e.g. [40]. We also checked that $\operatorname{ker}(L)^{\perp} \cap S_{x^{\star}}^{J}=\{0\}$, which is equivalent to the uniqueness of the fixed point and also implies that $M^{\infty}=0$ (see Lemma 6.2(i)). Thus (ND) is fulfilled, and Theorem 6.1 applies. DR is run in its stationary version (i.e. constant $\gamma=1 / 2$ ).

Figure 1 displays the profile of $\left\|z_{k}-z^{\star}\right\|$ as a function of $k$, and the starting point of the dashed line is the iteration number at which the active partial smoothness manifold of $J$ is identified (recall that $\mathcal{M}_{x^{\star}}^{G}=C$ which is trivially identified from the first iteration). One can easily see that for the $\ell_{1}$ and $\ell_{\infty}$ norms, the observed linear convergence coincides with the optimal rate predicted by Theorem 6.1(ii). For the case of $\ell_{1,2}$-norm and nuclear norm, though not optimal, our estimates are very tight.

\section{Noise removal}

In the following two examples, we suppose that we observe $y=x_{\mathrm{ob}}+\varepsilon$, where $x_{\mathrm{ob}}$ is a piecewise-constant vector, and $\varepsilon$ is an unknown noise supposed to be either uniform or sparse. The goal is to recover $x_{\mathrm{ob}}$ from $y$ using the prior information on $x_{\mathrm{ob}}$ (i.e. piecewise-smooth) and $\varepsilon$ (uniform or sparse). To achieve this goal, a popular and natural approach in the signal processing literature is to solve

$$
\min _{x \in \mathbb{R}^{n}}\|x\|_{\mathrm{TV}} \quad \text { subject to }\|y-x\|_{p} \leq \tau,
$$

where $p=+\infty$ for uniform noise, and $p=1$ for sparse noise, and $\tau>0$ is a parameter to be set by the user to adapt to the noise level. Identifying $J=\|\cdot\|_{\mathrm{TV}}$ and 


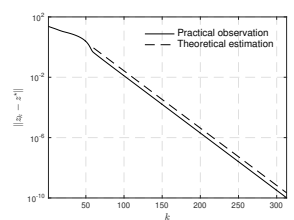

(a) $\ell_{1}$-norm

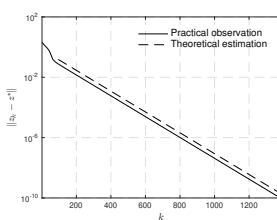

(b) $\ell_{\infty}$-norm

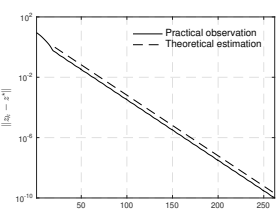

(c) $\ell_{1,2}$-norm

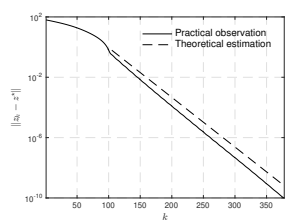

(d) Nuclear norm

Fig. 1 Observed (solid) and predicted (dashed) convergence profiles of DR (3) in terms of $\left\|z_{k}-z^{\star}\right\|$ with $\gamma_{k} \equiv 1 / 2$. (a) $\ell_{1}$-norm. (b) $\ell_{\infty}$-norm. (c) $\ell_{1,2}$-norm. (d) Nuclear norm. The starting point of the dashed line is the iteration at which the active manifold of $J$ is identified.

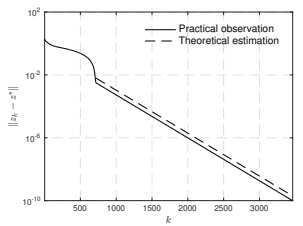

(a) Uniform noise removal

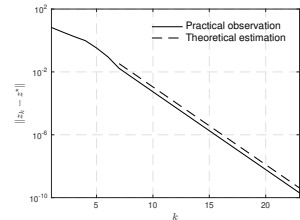

(b) Sparse noise removal

Fig. 2 Observed (solid) and predicted (dashed) convergence profiles of DR (3) in terms of $\left\|z_{k}-z^{\star}\right\|$ with $\gamma_{k} \equiv 1 / 2$. (a) Uniform noise removal by solving (23) with $p=+\infty$, (c) Sparse noise removal by solving (23) with $p=1$. The starting point of the dashed line is the iteration at which the manifolds $\mathcal{M}_{x^{\star}}^{J}$ and $\mathcal{M}_{x}^{G}$ are identified.

$G=\iota_{\|y-\cdot\|_{p} \leq \tau}$, one recognises that for $p \in\{1,+\infty\}, J$ and $G$ are indeed polyhedral and their proximity operators are simple to compute. For both examples, we set $n=128$ and $x_{\mathrm{ob}}$ is such that $D_{\mathrm{DIF}} x_{\mathrm{ob}}$ has 8 nonzero entries. For $p=+\infty, \varepsilon$ is generated uniformly in $[-1,1]$, and for $p=1 \varepsilon$ is sparse with 16 nonzero entries. DR is run in its stationary version. The corresponding local convergence profiles are depicted in Figure 2(a)-(b). Condition (ND) is checked posterior, and it is satisfied for the considered examples. Owing to polyhedrality, our rate predictions are again optimal.

\subsection{Finite Convergence}

We now numerically illustrate the finite convergence of DR. For the remainder of this subsection, we set $n=2$, and solve $(\mathcal{P})$ with $G=\|\cdot\|_{1}$ and $J=\iota_{C}, C=$ $\left\{x \in \mathbb{R}^{2}:\left\|x-(3 / 43 / 4)^{T}\right\|_{1} \leq 1 / 2\right\}$. The set of minimizers is the segment $\left[(1 / 43 / 4)^{T},(3 / 41 / 4)^{T}\right]$, and $G$ is differentiable at any minimizer with gradient $\left(\begin{array}{ll}1 & 1\end{array}\right)^{T}$. The set of fixed points is thus $\left[\left(\begin{array}{ll}1 / 4 & 3 / 4\end{array}\right)^{T},(3 / 41 / 4)^{T}\right]-\gamma$. Figure 3(a) shows the trajectory of the sequence $\left\{z_{k}\right\}_{k \in \mathbb{N}}$ and the shadow sequence $\left\{x_{k}\right\}_{k \in \mathbb{N}}$ which both converge finitely as predicted by Theorem 7.1 (DR is used with $\gamma=0.25$ ).

For each starting point $z_{0} \in[-10,10]^{2}$, we run the DR algorithm until $z_{k+1}=z_{k}$ (up to machine precision), with $\gamma=0.25$ and $\gamma=5$. Figure 3(b)-(c) show the number of iterations to finite convergence, where $\gamma=0.25$ for (b) and $\gamma=5$ for (c). This confirms that DR indeed converges in finitely many iterations regardless of the starting 


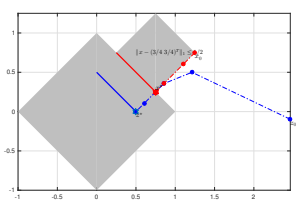

(a)

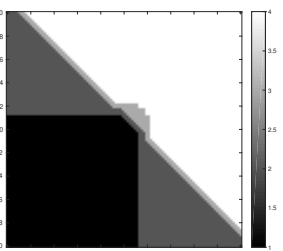

(b) $\gamma=0.25$

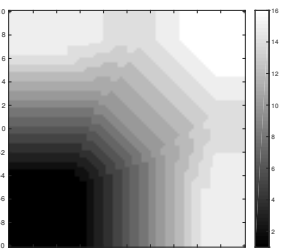

(c) $\gamma=5$

Fig. 3 (a) Trajectories of $\left\{z_{k}\right\}_{k \in \mathbb{N}}$ and $\left\{x_{k}\right\}_{k \in \mathbb{N}}$. The red segment is the set of minimizers and the blue one is the set of fixed points. (b)-(c) Number of iterations needed for the finite convergence of $z_{k}$ to $z^{\star}$. DR is run with $\gamma=0.25$ for (b) and $\gamma=5$ for (c).

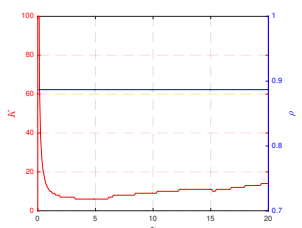

(a) $\ell_{1}$-norm

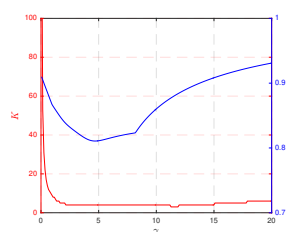

(b) $\ell_{1,2}$-norm

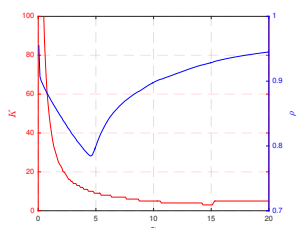

(c) Nuclear norm

Fig. 4 Number of iterations $(K)$ needed for identification and local linear convergence rate $(\rho)$ as a function of $\gamma$ when solving problem (22) with different functions $J$ in Table 1 . (a) $\ell_{1}$-norm. (b) $\ell_{1,2}$-norm. (c) Nuclear norm.

point and choice of $\gamma$, though more iterations are needed for higher $\gamma$ in this example (see next subsection for further discussion on the choice of $\gamma$ ).

\subsection{Choice of $\gamma$}

\section{Impact of $\gamma$ on identification}

We now turn to the impact of the choice of $\gamma$ in the DR algorithm. We consider (22) with $J$ being the $\ell_{1}$, the $\ell_{1,2}$ and nuclear norms.

The results are shown in Figure 4, where $K$ denotes the number of iterations needed to identify $\mathcal{M}_{x^{\star}}^{J}$ and $\rho$ denotes the local linear convergence rate. We summarize our observations as follows:

- For all examples, the choice of $\gamma$ affects the iteration $K$ at which activity identification occurs. Indeed, $K$ typically decreases monotonically and then either stabilizes or slightly increases. This is in agreement with the bound in (8);

- When $J$ is the $\ell_{1}$, which is polyhedral, the local linear convergence rate is insensitive to $\gamma$ as anticipated by Theorem 6.1(ii). For the other two norms, the local rate depends on $\gamma$ (see Theorem 6.1(i)), and this rate can be optimized for the parameter $\gamma$;

- In general, there is no correspondence between the optimal choice of $\gamma$ for identification and the one for local convergence rate. 


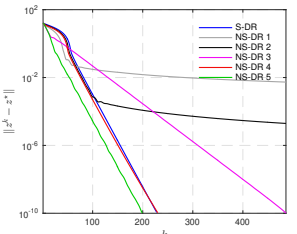

(a) $\ell_{1}$-norm

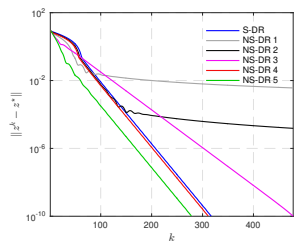

(b) $\ell_{1,2}$-norm

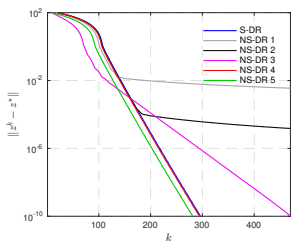

(c) Nuclear norm

Fig. 5 Comparison between stationary ("S-DR") and non-stationary DR ("NS-DR X", X stands for Case $\mathrm{X}$ ) when solving (22) with different functions $J$ in Table 1. (a) $\ell_{1}$-norm. (b) $\ell_{1,2}$-norm. (c) Nuclear norm.

Table 2 Number of iterations $K$ needed for the identification of $\mathcal{M}_{x^{\star}}^{J}$ for each tested case. "NS-DR X" stands for the non-stationary DR with choice of $\gamma_{k}$ as in Case X.

\begin{tabular}{|c|c|c|c|c|c|c|}
\hline & S-DR & NS-DR 1 & NS-DR 2 & NS-DR 3 & NS-DR 4 & NS-DR 5 \\
\hline$\ell_{1}$-norm & 62 & 46 & 59 & 244 & 56 & 109 \\
\hline$\ell_{1,2}$-norm & 47 & 42 & 43 & 227 & 41 & 79 \\
\hline Nuclear norm & 109 & 103 & 108 & 72 & 107 & 94 \\
\hline
\end{tabular}

\section{Stationary vs non-stationary DR}

We now investigate numerically the convergence behaviour of the non-stationary version of DR and compate it to the stationary one. We fix $\lambda_{k} \equiv 1$, i.e. the iteration is unrelaxed. The stationary DR algorithm is run with some $\gamma>0$. For the non-stationary one, four choices of $\gamma_{k}$ are considered:

$$
\begin{aligned}
& \text { Case 1: } \gamma_{k}=\gamma+\frac{1}{k^{1.1}}, \text { Case 2: } \gamma_{k}=\gamma+\frac{1}{k^{2}}, \text { Case 3: } \gamma_{k}=\gamma+0.95^{k}, \\
& \text { Case 4: } \gamma_{k}=\gamma+0.5^{k}, \text { Case 5: } \gamma_{k}=\gamma+e^{-k / 8} .
\end{aligned}
$$

Obviously, we have $\left\{\left|\gamma_{k}-\gamma\right|\right\}_{k \in \mathbb{N}} \in \ell_{+}^{1}$ for all the four cases. Problem (22) is considered again with $J$ the $\ell_{1}$, the $\ell_{1,2}$ and the nuclear norms. The comparison results are displayed in Figure 5. Table 2 shows the number of iteration $K$ needed for the identification of $\mathcal{M}_{x^{\star}}^{J}$.

For the stationary iteration, the local convergence rate of the 3 examples are,

$\ell_{1}$-norm: $\rho=0.9129, \quad \ell_{1,2}$-norm: $\rho=0.9324, \quad$ Nuclear norm: $\rho=0.8869$.

We can make the following observations from the comparison:

- The local convergence behvaiour of the non-stationary iteration is no better than the stationary one (same local convergence rate) which is in agreement with our analysis;

- As argued in Remark 6.2(ii), the convergence rate is eventually controlled by the error $\left|\gamma_{k}-\gamma\right|$, except for "Case $4 \& 5$ ", since 0.5 and the exponential function decay faster than the local linear rate of the stationary version (i.e. $\left|\gamma_{k}-\gamma\right|=$ $\left.o\left(\left\|z_{k}-z^{\star}\right\|\right)\right)$

- The non-stationary DR seems to generally lead to faster identification. Though this is not a systematic behaviour as observed for instance for "Case $3 \& 5$ ", where slower identification is obtained for the $\ell_{1}$ and the $\ell_{1,2}$ norms.

Overall, "Case 5" shows the best performance, which implies that in practice, at least for the presented examples, a good strategy is use bigger values of $\gamma_{k}$ at beginning for 
a faster identification, and locally converges to the limit value quickly in order to have faster local performance.

\section{Conclusions}

In this paper, we investigated local convergence properties of DR and ADMM when the involved functions (or their conjugates) are convex and partly smooth. In particular, we showed that these schemes identify the active manifolds in finite time and then converge locally linearly at a rate that we characterized precisely. Under polyhedrality of both functions, we also characterize situations where finite convergence occurs. Future work includes several extensions of this work. At first, finite identification and finite convergence under milder assumptions than those required here would be important. Another important extension would be to tackle the non-convex setting.

\section{Appendices}

\section{Appendix A}

We start with the following lemma which is needed in the proof of Theorem 4.1.

Lemma A.1 Suppose that conditions (H.2) and (H.3) hold, and that $\gamma_{k}$ is convergent. Then

$$
\lim _{k \rightarrow+\infty} \gamma_{k}=\gamma
$$

Proof Since $\gamma_{k}$ is convergent, it has a unique cluster point, say $\lim _{k \rightarrow+\infty} \gamma_{k}=\gamma^{\prime}$. It is then sufficient to show that $\gamma^{\prime}=\gamma$. Suppose that $\gamma^{\prime} \neq \gamma$. Fix some $\left.\varepsilon \in\right] 0,\left|\gamma^{\prime}-\gamma\right|[$. Thus, there exist an index $K>0$ such that for all $k \geq K$,

$$
\left|\gamma_{k}-\gamma^{\prime}\right|<\varepsilon / 2 \text {. }
$$

Therefore

It then follows that

$$
\left|\gamma_{k}-\gamma\right| \geq\left|\gamma^{\prime}-\gamma\right|-\left|\gamma_{k}-\gamma^{\prime}\right|>\varepsilon / 2
$$

$$
\lambda_{k}\left(2-\lambda_{k}\right) \varepsilon \leq 2 \lambda_{k} \varepsilon \leq 4 \lambda_{k}\left|\gamma_{k}-\gamma\right|
$$

Denote $\bar{\tau}:=\sup _{k \mathbb{N}} \lambda_{k}\left(2-\lambda_{k}\right)$ which is obviously positive and bounded since $\lambda_{k} \in[0,2]$. Summing both sides for $k \geq K$ we get

$$
\varepsilon \sum_{k \in \mathbb{N}} \lambda_{k}\left(2-\lambda_{k}\right)-K \bar{\tau} \leq \varepsilon \sum_{k=K}^{+\infty} \lambda_{k}\left(2-\lambda_{k}\right) \leq 4 \sum_{k \in \mathbb{N}} \lambda_{k}\left|\gamma_{k}-\gamma\right|,
$$

which, in view of (H.3), implies

$$
\sum_{k \in \mathbb{N}} \lambda_{k}\left(2-\lambda_{k}\right) \leq \varepsilon^{-1}\left(\lambda_{k}\left|\gamma_{k}-\gamma\right|+K \bar{\tau}\right)<+\infty,
$$

leading to a contradiction with (H.2).

Proof (Theorem 4.1) To prove our claim, we only need to check the conditions listed in [3, Theorem 4].

(i) As (A.3) assumes the set of minimizers of $(\mathcal{P})$ is nonempty, so is the set $\operatorname{Fix}\left(\mathscr{F}_{\gamma}\right)$, since the former is nothing but $\operatorname{prox}_{\gamma J}\left(\operatorname{Fix}\left(\mathscr{F}_{\gamma}\right)\right)$ [20, Proposition 25.1(ii)].

(ii) Since $\mathscr{F}_{\gamma_{k}}$ is firmly nonexpansive by Lemma $2.2, \mathscr{F}_{\gamma_{k}, \lambda_{k}}$ is $\frac{\lambda_{k}}{2}$-averaged nonexpansive, hence nonexpansive, owing to Lemma 2.1(iv). 
(iii) Let $\rho \in\left[0,+\infty\left[\right.\right.$ and $z \in \mathbb{R}^{n}$ such that $\|z\| \leq \rho$, Then we have

$$
\begin{aligned}
\left(\mathscr{F}_{\gamma_{k}}-\mathscr{F}_{\gamma}\right)(z) & =\frac{\operatorname{rprox}_{\gamma_{k} G} \operatorname{orprox}_{\gamma_{k} J}}{2}(z)-\frac{\operatorname{rprox}_{\gamma G} \operatorname{orprox}_{\gamma J}}{2}(z) \\
& =\left(\frac{\operatorname{rprox}_{\gamma_{k} G} \operatorname{orprox}_{\gamma_{k} J}}{2}(z)-\frac{\operatorname{rprox}_{\gamma_{k} G} \operatorname{orprox}_{\gamma J}}{2}(z)\right) \\
& -\left(\frac{\operatorname{rprox}_{\gamma G} \operatorname{orprox}_{\gamma J}}{2}(z)-\frac{\operatorname{rprox}_{\gamma_{k} G} \operatorname{orprox}_{\gamma J}}{2}(z)\right) \\
& =\left(\frac{\operatorname{rprox}_{\gamma_{k} G} \operatorname{orprox}_{\gamma_{k}} J}{2}(z)-\frac{\operatorname{rprox}_{\gamma_{k} G} \operatorname{orprox}_{\gamma J}}{2}(z)\right) \\
& -\left(\operatorname{prox}_{\gamma G} \circ \operatorname{rprox}_{\gamma J}(z)-\operatorname{prox}_{\gamma_{k} G} \circ \operatorname{rprox}_{\gamma J}(z)\right) .
\end{aligned}
$$

Thus, by virtue of Lemma 2.1(iii), we have

$$
\begin{aligned}
& \left\|\left(\mathscr{F}_{\gamma_{k}}-\mathscr{F}_{\gamma}\right)(z)\right\| \\
& \leq\left\|\operatorname{prox}_{\gamma_{k} J}(z)-\operatorname{prox}_{\gamma J}(z)\right\|+\left\|\operatorname{prox}_{\gamma_{k} G}\left(\operatorname{pprox}_{\gamma J}(z)\right)-\operatorname{prox}_{\gamma G}\left(\operatorname{prox}_{\gamma J}(z)\right)\right\| .
\end{aligned}
$$

Let's bound the first term. From the resolvent equation [41], and Lemma 2.1(i)(ii)(v), we have

$$
\begin{aligned}
\left\|\operatorname{prox}_{\gamma_{k} J}(z)-\operatorname{prox}_{\gamma J}(z)\right\| & =\left\|\operatorname{prox}_{\gamma_{k} J}(z)-\operatorname{prox}_{\gamma_{k} J}\left(\frac{\gamma_{k}}{\gamma} z+\left(1-\frac{\gamma_{k}}{\gamma}\right) \operatorname{prox}_{\gamma J}(z)\right)\right\| \\
& \leq \frac{\left|\gamma_{k}-\gamma\right|}{\gamma}\left\|\left(\operatorname{Id}-\operatorname{prox}_{\gamma J}\right)(z)\right\| \leq \frac{\left|\gamma_{k}-\gamma\right|}{\gamma}\left(\rho+\left\|\operatorname{prox}_{\gamma J}(0)\right\|\right) .
\end{aligned}
$$

With similar arguments, we also obtain

$\left.\left\|\operatorname{prox}_{\gamma_{k} G}\left(\operatorname{rprox}_{\gamma J}(z)\right)-\operatorname{prox}_{\gamma G}\left(\operatorname{rprox}_{\gamma J}(z)\right)\right\| \leq \frac{\left|\gamma_{k}-\gamma\right|}{\gamma}\left(\rho+\left\|\operatorname{prox}_{\gamma G}(0)\right\|+2 \| \operatorname{prox}_{\gamma J}(0)\right) \|\right)$.

Combining (24) and (25) leads to

$$
\left\|\left(\mathscr{F}_{\gamma_{k}}-\mathscr{F}_{\gamma}\right)(z)\right\| \leq \frac{\left|\gamma_{k}-\gamma\right|}{\gamma}\left(2 \rho+\left\|\operatorname{prox}_{\gamma G}(0)\right\|+3\left\|\operatorname{prox}_{\gamma J}(0)\right\|\right),
$$

whence we get

$$
\begin{aligned}
& \left\|\left(\mathscr{F}_{\gamma_{k}, \lambda_{k}}-\mathscr{F}_{\gamma, \lambda_{k}}\right)(z)\right\|=\lambda_{k}\left\|\left(\mathscr{F}_{\gamma_{k}}-\mathscr{F}_{\gamma}\right)(z)\right\| \leq \lambda_{k} \frac{\left|\gamma_{k}-\gamma\right|}{\gamma}\left(2 \rho+\left\|\operatorname{prox}_{\gamma G}(0)\right\|\right. \\
& \left.+3\left\|\operatorname{prox}_{\gamma J}(0)\right\|\right) \text {. }
\end{aligned}
$$

Therefore, from (H.3), we deduce that

$$
\left\{\sup _{\|z\| \leq \rho}\left\|\left(\mathscr{F}_{\gamma_{k}, \lambda_{k}}-\mathscr{F}_{\gamma, \lambda_{k}}\right)(z)\right\|\right\}_{k \in \mathbb{N}} \in \ell_{+}^{1} .
$$

In other words, the non-stationary iteration (7) is a perturbed version of the stationary one (4) with an error term which is summable thanks to (H.3). The claim on the convergence of $z^{\star}$ follows by applying [24, Corollary 5.2]. Moreover, $x^{\star}:=\operatorname{prox}_{\gamma J}\left(z^{\star}\right)$ is a solution of $(\mathcal{P})$. In turn, using nonexpansiveness of $\operatorname{prox}_{\gamma_{k} J}$ and (24), we have

$$
\left\|x_{k}-x^{\star}\right\| \leq\left\|z_{k}-z^{\star}\right\|+\frac{\left|\gamma_{k}-\gamma\right|}{\gamma}\left(\left\|z^{\star}\right\|+\left\|\operatorname{prox}_{\gamma J}(0)\right\|\right),
$$

and thus the right hand side goes to zero as $k \rightarrow+\infty$ as we are in finite dimension and since $\gamma_{k} \rightarrow \gamma$ owing to Lemma A.1. This entails that the shadow sequence $\left\{x_{k}\right\}_{k \in \mathbb{N}}$ also converges to $x^{\star}$. With similar arguments, we can also show that $\left\{v_{k}\right\}_{k \in \mathbb{N}}$ converges to $x^{\star}$ (using for instance (25) and nonexpansiveness of $\operatorname{prox}_{\gamma_{k} G}$ ). 


\section{Appendix B}

Proof (Theorem 5.1) By Theorem 4.1, all the sequences generated by (6) converge, i.e.

$$
z_{k} \rightarrow z^{\star} \in \operatorname{Fix}\left(\mathscr{F}_{\gamma, \lambda}\right), x_{k}, v_{k}, \rightarrow x^{\star}=\operatorname{prox}_{\gamma}\left(z^{\star}\right) \in \operatorname{Argmin}(G+J) .
$$

The nondegeneracy condition (ND) is equivalent to

$$
\frac{x^{\star}-z^{\star}}{\gamma} \in \operatorname{ri}\left(\partial G\left(x^{\star}\right)\right) \text { and } \frac{z^{\star}-x^{\star}}{\gamma} \in \operatorname{ri}\left(\partial J\left(x^{\star}\right)\right) .
$$

(i) The update of $x_{k+1}$ and $v_{k+1}$ in iteration (6) is equivalent to the monotone inclusions

$$
\frac{2 x_{k}-z_{k}-v_{k+1}}{\gamma_{k}} \in \partial G\left(v_{k+1}\right) \text { and } \frac{z_{k}-x_{k}}{\gamma_{k}} \in \partial J\left(x_{k}\right) \text {. }
$$

It then follows that

$$
\begin{aligned}
& \operatorname{dist}\left(\frac{x^{\star}-z^{\star}}{\gamma}, \partial G\left(v_{k+1}\right)\right) \leq\left\|\frac{x^{\star}-z^{\star}}{\gamma}-\frac{2 x_{k}-z_{k}-v_{k+1}}{\gamma_{k}}\right\| \\
&=\left\|\frac{\left(\gamma_{k}-\gamma\right)\left(x^{\star}-z^{\star}\right)}{\gamma \gamma_{k}}+\frac{x^{\star}-z^{\star}}{\gamma_{k}}-\frac{2 x_{k}-z_{k}-v_{k+1} \|}{\gamma_{k}}\right\| \\
& \leq \frac{\left|\gamma_{k}-\gamma\right|}{\gamma \underline{\gamma}}\left\|\left(\mathrm{Id}-\operatorname{prox}_{\gamma J}\right)\left(z^{\star}\right)\right\|+\frac{1}{\underline{\gamma}} \|\left(z_{k}-z^{\star}\right)-2\left(x_{k}-x^{\star}\right) \\
& \leq \frac{\left|\gamma_{k}-\gamma\right|}{\gamma \underline{\gamma}}\left(\left\|z^{\star}\right\|+\operatorname{prox}_{\gamma J}(0)\right)+\frac{1}{\underline{\gamma}}\left(\left\|z_{k}-z^{\star}\right\|+2\left\|x_{k}-x^{\star}\right\|\right. \\
&\left.+\left\|v_{k+1}-x^{\star}\right\|\right),
\end{aligned}
$$

and the right hand side converges to 0 in view of Theorem 4.1 and Lemma A.1. Similarly, we have

$$
\begin{aligned}
\operatorname{dist}\left(\frac{z^{\star}-x^{\star}}{\gamma}, \partial J\left(x_{k}\right)\right) & \leq\left\|\frac{z^{\star}-x^{\star}}{\gamma}-\frac{z_{k}-x_{k}}{\gamma_{k}}\right\|=\| \frac{\left(\gamma_{k}-\gamma\right)\left(z^{\star}-x^{\star}\right)}{\gamma \gamma_{k}}+\frac{z^{\star}-x^{\star}}{\gamma_{k}} \\
& \leq \frac{\left|\gamma_{k}-\gamma\right|}{\gamma \underline{\gamma}}\left(\left\|z^{\star}\right\|+\operatorname{prox}_{\gamma J}(0)\right)+\frac{1}{\underline{\gamma}}\left(\left\|z_{k}-z^{\star}\right\|+\left\|x_{k}-x^{\star}\right\|\right) \rightarrow 0 .
\end{aligned}
$$

By assumption, $G, J \in \Gamma_{0}\left(\mathbb{R}^{n}\right)$, hence are subdifferentially continuous at every point in their respective domains [32, Example 13.30], and in particular at $x^{\star}$. It then follows that $G\left(v_{k}\right) \rightarrow G\left(x^{\star}\right)$ and $J\left(x_{k}\right) \rightarrow J\left(x^{\star}\right)$. Altogether, this shows that the conditions of [42, Theorem 5.3] are fulfilled for $G$ and $J$, and the finite identification claim follows.

(ii) (a) In this case, $\mathcal{M}_{x^{\star}}^{J}$ is an affine subspace, i.e. $\mathcal{M}_{x^{\star}}^{J}=x^{\star}+T_{x^{\star}}^{J}$. Since $J$ is partly smooth at $x^{\star}$ relative to $\mathcal{M}_{x^{\star}}^{J}$, the sharpness property holds at all nearby points in $\mathcal{M}_{x^{\star}}^{J}[$, Proposition 2.10]. Thus for $k$ large enough, i.e. $x_{k}$ sufficiently close to $x^{\star}$ on $\mathcal{M}_{x^{\star}}^{J}$, we have indeed $\mathcal{T}_{x_{k}}\left(\mathcal{M}_{x^{\star}}^{J}\right)=T_{x^{\star}}^{J}=T_{x_{k}}^{J}$ as claimed.

(b) Similar to (ii)(a)

(c) It is immediate to verify that a locally polyhedral function around $x^{\star}$ is indeed partly smooth relative to the affine subspace $x^{\star}+T_{x^{\star}}^{J}$, and thus, the first claim follows from (ii)(a). For the rest, it is sufficient to observe that by polyhedrality, for any $x \in \mathcal{M}_{x^{\star}}^{J}$ near $x^{\star}$, $\partial J(x)=\partial J\left(x^{\star}\right)$. Therefore, combining local normal sharpness [9, Proposition 2.10] and Lemma 5.1 yields the second conclusion.

(d) Similar to (ii)(c).

Proof (Proposition 5.1) From (7), we have

$$
z_{k+1}=\mathscr{F}_{\gamma, \lambda_{k}}\left(z_{k}\right)+e_{k}
$$


where $\left\{\left\|e_{k}\right\|\right\}_{k \in \mathbb{N}}=\left\{O\left(\lambda_{k}\left|\gamma_{k}-\gamma\right|\right)\right\}_{k \in \mathbb{N}} \in \ell_{+}^{1}$ (see the proof of Theorem 4.1). Since $\mathscr{F}_{\gamma_{k}}$ is firmly non-expansive by Lemma 2.2, $\mathscr{F}_{\gamma, \lambda_{k}}$ is $\frac{\lambda_{k}}{2}$-averaged non-expansive owing to Lemma 2.1(iv). Thus arguing as in the proof of [24, Theorem 3.1], we have

$$
\begin{aligned}
\left\|z_{k}-z^{\star}\right\|^{2} & \leq\left\|\mathscr{F}_{\gamma, \lambda_{k}}\left(z_{k-1}\right)-\mathscr{F}_{\gamma, \lambda_{k}}\left(z^{\star}\right)\right\|^{2}+C\left\|e_{k-1}\right\| \\
& \leq\left\|\mathscr{F}_{\gamma, \lambda_{k}}\left(z_{k-1}\right)-\mathscr{F}_{\gamma, \lambda_{k}}\left(z^{\star}\right)\right\|^{2}-\frac{2-\lambda_{k-1}}{\lambda_{k-1}}\left\|z_{k}-z_{k-1}\right\|^{2}+C\left\|e_{k-1}\right\| \\
& \leq\left\|z_{k-1}-z^{\star}\right\|^{2}-\tau_{k-1}\left\|v_{k}-x_{k-1}\right\|^{2}+C\left\|e_{k-1}\right\|,
\end{aligned}
$$

where $C<+\infty$ by boundedness of $z_{k}$ and $e_{k}$. Let $g_{k}=\left(z_{k-1}-x_{k-1}\right) / \gamma_{k-1}$ and $h_{k}=\left(2 x_{k-1}-z_{k-1}-v_{k}\right) / \gamma_{k-1}$. By definition, we have $\left(g_{k}, h_{k}\right) \in \partial J\left(x_{k-1}\right) \times \partial G\left(v_{k}\right)$. Suppose that neither $\mathcal{M}_{x^{\star}}^{J}$ nor $\mathcal{M}_{x^{\star}}^{G}$ have been identified at iteration $k$. That is $x_{k-1} \notin \mathcal{M}_{x^{\star}}^{J}$ and $v_{k} \notin \mathcal{M}_{x^{\star}}^{G}$, and by assumption, $g_{k} \in \operatorname{rbd}\left(\partial J\left(x^{\star}\right)\right)$ and $h_{k} \in \operatorname{rbd}\left(\partial G\left(x^{\star}\right)\right)$, which implies that $g_{k}+h_{k}=\left(v_{k}-x_{k-1}\right) / \gamma_{k-1} \in \operatorname{rbd}\left(\partial J\left(x^{\star}\right)\right)+\operatorname{rbd}\left(\partial G\left(x^{\star}\right)\right)$. Thus, the above inequality becomes

$$
\begin{aligned}
\left\|z_{k}-z^{\star}\right\|^{2} & \leq\left\|z_{k-1}-z^{\star}\right\|^{2}-\gamma_{k-1}^{2} \tau_{k-1} \operatorname{dist}\left(0, \operatorname{rbd}\left(\partial J\left(x^{\star}\right)\right)+\operatorname{rbd}\left(\partial G\left(x^{\star}\right)\right)\right)^{2}+C\left\|e_{k-1}\right\| \\
& \leq\left\|z_{k-1}-z^{\star}\right\|^{2}-\gamma_{k-1}^{2} \tau_{k-1} \operatorname{dist}\left(0, \operatorname{rbd}\left(\partial J\left(x^{\star}\right)+\partial G\left(x^{\star}\right)\right)\right)^{2}+C\left\|e_{k-1}\right\| \\
& \leq\left\|z_{0}-z^{\star}\right\|^{2}-k \underline{\gamma}^{2} \underline{\tau} \operatorname{dist}\left(0, \operatorname{rbd}\left(\partial J\left(x^{\star}\right)+\partial G\left(x^{\star}\right)\right)\right)^{2}+O\left(\sum_{k \in \mathbb{N}} \lambda_{k}\left|\gamma_{k}-\gamma\right|\right),
\end{aligned}
$$

and $\operatorname{dist}\left(0, \operatorname{rbd}\left(\partial J\left(x^{\star}\right)+\partial G\left(x^{\star}\right)\right)\right)>0$ owing to condition (ND). Taking $k$ as the largest integer such that the bound in the right hand is positive, we deduce that the number of iterations where both $\mathcal{M}_{x^{\star}}^{J}$ and $\mathcal{M}_{x^{\star}}^{G}$ have not been identified yet does not exceed the claimed bound (8). Thus finite identification necessarily occurs at some $k$ larger than this bound.

\section{Appendix C}

\section{Riemannian Geometry}

Let $\mathcal{M}$ be a $C^{2}$-smooth embedded submanifold of $\mathbb{R}^{n}$ around a point $x$. With some abuse of terminology, we shall state $C^{2}$-manifold instead of $C^{2}$-smooth embedded submanifold of $\mathbb{R}^{n}$. The natural embedding of a submanifold $\mathcal{M}$ into $\mathbb{R}^{n}$ permits to define a Riemannian structure and to introduce geodesics on $\mathcal{M}$, and we simply say $\mathcal{M}$ is a Riemannian manifold. We denote respectively $\mathcal{T}_{\mathcal{M}}(x)$ and $\mathcal{N}_{\mathcal{M}}(x)$ the tangent and normal space of $\mathcal{M}$ at point near $x$ in $\mathcal{M}$.

\section{Exponential map}

Geodesics generalize the concept of straight lines in $\mathbb{R}^{n}$, preserving the zero acceleration characteristic, to manifolds. Roughly speaking, a geodesic is locally the shortest path between two points on $\mathcal{M}$. We denote by $\mathfrak{g}(t ; x, h)$ the value at $t \in \mathbb{R}$ of the geodesic starting at $\mathfrak{g}(0 ; x, h)=x \in \mathcal{M}$ with velocity $\dot{\mathfrak{g}}(t ; x, h)=\frac{d \mathfrak{g}}{d t}(t ; x, h)=h \in \mathcal{T}_{\mathcal{M}}(x)$ (which is uniquely defined). For every $h \in \mathcal{T}_{\mathcal{M}}(x)$, there exists an interval $I$ around 0 and a unique geodesic $\mathfrak{g}(t ; x, h): I \rightarrow \mathcal{M}$ such that $\mathfrak{g}(0 ; x, h)=x$ and $\dot{\mathfrak{g}}(0 ; x, h)=h$. The mapping

$$
\operatorname{Exp}_{x}: \mathcal{T}_{\mathcal{M}}(x) \rightarrow \mathcal{M}, h \mapsto \operatorname{Exp}_{x}(h)=\mathfrak{g}(1 ; x, h),
$$

is called Exponential map. Given $x, x^{\prime} \in \mathcal{M}$, the direction $h \in \mathcal{T}_{\mathcal{M}}(x)$ we are interested in is such that $\operatorname{Exp}_{x}(h)=x^{\prime}=\mathfrak{g}(1 ; x, h)$.

\section{Parallel translation}

Given two points $x, x^{\prime} \in \mathcal{M}$, let $\mathcal{T}_{\mathcal{M}}(x), \mathcal{T}_{\mathcal{M}}\left(x^{\prime}\right)$ be their corresponding tangent spaces. Define $\tau$ : $\mathcal{T}_{\mathcal{M}}(x) \rightarrow \mathcal{T}_{\mathcal{M}}\left(x^{\prime}\right)$ the parallel translation along the unique geodesic joining $x$ to $x^{\prime}$, which is isomorphism and isometry w.r.t. the Riemannian metric. 
Riemannian gradient and Hessian

For a vector $v \in \mathcal{N}_{\mathcal{M}}(x)$, the Weingarten map of $\mathcal{M}$ at $x$ is the operator $\mathfrak{W}_{x}(\cdot, v): \mathcal{T}_{\mathcal{M}}(x) \rightarrow \mathcal{T}_{\mathcal{M}}(x)$ defined by $\mathfrak{W}_{x}(\cdot, v)=-\mathrm{P}_{\mathcal{T}_{\mathcal{M}}(x)} \mathrm{d} V[h]$, where $V$ is any local extension of $v$ to a normal vector field on $\mathcal{M}$. The definition is independent of the choice of the extension $V$, and $\mathfrak{W}_{x}(\cdot, v)$ is a symmetric linear operator which is closely tied to the second fundamental form of $\mathcal{M}$, see [43, Proposition II.2.1].

Let $G$ be a real-valued function which is $C^{2}$ along the $\mathcal{M}$ around $x$. The covariant gradient of $G$ at $x^{\prime} \in \mathcal{M}$ is the vector $\nabla_{\mathcal{M}} G\left(x^{\prime}\right) \in \mathcal{T}_{\mathcal{M}}\left(x^{\prime}\right)$ defined by

$$
\left\langle\nabla_{\mathcal{M}} G\left(x^{\prime}\right), h\right\rangle=\left.\frac{d}{d t} G\left(\mathrm{P}_{\mathcal{M}}\left(x^{\prime}+t h\right)\right)\right|_{t=0}, \forall h \in \mathcal{T}_{\mathcal{M}}\left(x^{\prime}\right)
$$

where $\mathrm{P}_{\mathcal{M}}$ is the projection operator onto $\mathcal{M}$. The covariant Hessian of $G$ at $x^{\prime}$ is the symmetric linear mapping $\nabla_{\mathcal{M}}^{2} G\left(x^{\prime}\right)$ from $\mathcal{T}_{\mathcal{M}}\left(x^{\prime}\right)$ to itself which is defined as

$$
\left\langle\nabla_{\mathcal{M}}^{2} G\left(x^{\prime}\right) h, h\right\rangle=\left.\frac{d^{2}}{d t^{2}} G\left(\mathrm{P}_{\mathcal{M}}\left(x^{\prime}+t h\right)\right)\right|_{t=0}, \forall h \in \mathcal{T}_{\mathcal{M}}\left(x^{\prime}\right) .
$$

This definition agrees with the usual definition using geodesics or connections [44]. Now assume that $\mathcal{M}$ is a Riemannian embedded submanifold of $\mathbb{R}^{n}$, and that a function $G$ has a $C^{2}$-smooth restriction on $\mathcal{M}$. This can be characterized by the existence of a $C^{2}$-smooth extension (representative) of $G$, i.e. a $C^{2}$-smooth function $\widetilde{G}$ on $\mathbb{R}^{n}$ such that $\widetilde{G}$ agrees with $G$ on $\mathcal{M}$. Thus, the Riemannian gradient $\nabla_{\mathcal{M}} G\left(x^{\prime}\right)$ is also given by

$$
\nabla_{\mathcal{M}} G\left(x^{\prime}\right)=\mathrm{P}_{\mathcal{T}_{\mathcal{M}}\left(x^{\prime}\right)} \nabla \widetilde{G}\left(x^{\prime}\right)
$$

and $\forall h \in \mathcal{T}_{\mathcal{M}}\left(x^{\prime}\right)$, the Riemannian Hessian reads

$$
\begin{aligned}
\nabla_{\mathcal{M}}^{2} G\left(x^{\prime}\right) h & =\mathrm{P}_{\mathcal{T}_{\mathcal{M}}\left(x^{\prime}\right)} \mathrm{d}\left(\nabla_{\mathcal{M}} G\right)\left(x^{\prime}\right)[h]=\mathrm{P}_{\mathcal{T}_{\mathcal{M}}\left(x^{\prime}\right)} \mathrm{d}\left(x^{\prime} \mapsto \mathrm{P}_{\mathcal{T}_{\mathcal{M}}\left(x^{\prime}\right)} \nabla_{\mathcal{M}} \widetilde{G}\right)[h] \\
& =\mathrm{P}_{\mathcal{T}_{\mathcal{M}}\left(x^{\prime}\right)} \nabla^{2} \widetilde{G}\left(x^{\prime}\right) h+\mathfrak{W}_{x^{\prime}}\left(h, \mathrm{P}_{\mathcal{N}_{\mathcal{M}}\left(x^{\prime}\right)} \nabla \widetilde{G}\left(x^{\prime}\right)\right),
\end{aligned}
$$

where the last equality comes from [45, Theorem 1]. When $\mathcal{M}$ is an affine or linear subspace of $\mathbb{R}^{n}$, then obviously $\mathcal{M}=x+\mathcal{T}_{\mathcal{M}}(x)$, and $\mathfrak{W}_{x^{\prime}}\left(h, \mathrm{P}_{\mathcal{N}_{\mathcal{M}}\left(x^{\prime}\right)} \nabla \widetilde{G}\left(x^{\prime}\right)\right)=0$, hence (30) reduces to $\nabla_{\mathcal{M}}^{2} G\left(x^{\prime}\right)=\mathrm{P}_{\mathcal{T}_{\mathcal{M}}\left(x^{\prime}\right)} \nabla^{2} \widetilde{G}\left(x^{\prime}\right) \mathrm{P}_{\mathcal{T}_{\mathcal{M}}\left(x^{\prime}\right)}$. See [46,43] for more materials on differential and Riemannian manifolds.

We have the following proposition characterising the parallel translation and the Riemannian Hessian of two close points in $\mathcal{M}$.

Lemma C.1 Let $x, x^{\prime}$ be two close points in $\mathcal{M}$, denote $\mathcal{T}_{\mathcal{M}}(x), \mathcal{T}_{\mathcal{M}}\left(x^{\prime}\right)$ be the tangent spaces of $\mathcal{M}$ at $x, x^{\prime}$ respectively, and $\tau: \mathcal{T}_{\mathcal{M}}\left(x^{\prime}\right) \rightarrow \mathcal{T}_{\mathcal{M}}(x)$ be the parallel translation along the unique geodesic joining from $x$ to $x^{\prime}$, then for the parallel translation we have, given any bounded vector $v \in \mathbb{R}^{n}$

$$
\left(\tau \mathrm{P}_{\mathcal{T}_{\mathcal{M}}\left(x^{\prime}\right)}-\mathrm{P}_{\mathcal{T}_{\mathcal{M}}(x)}\right) v=o(v) .
$$

The Riemannian Taylor expansion of $J \in C^{2}(\mathcal{M})$ at $x$ for $x^{\prime}$ reads,

$$
\tau \nabla_{\mathcal{M}} J\left(x^{\prime}\right)=\nabla_{\mathcal{M}} J(x)+\nabla_{\mathcal{M}}^{2} J(x) \mathrm{P}_{\mathcal{T}_{\mathcal{M}}(x)}\left(x^{\prime}-x\right)+o\left(x^{\prime}-x\right) .
$$

Proof See [30, Lemma B.1 and B.2].

Proof (Proposition 6.1) Since $W_{\bar{G}}, W_{\bar{J}}$ are both firmly non-expansive by Lemma 6.1, it follows from [20, Example 4.7] that $M_{\bar{G}}$ and $M_{\bar{J}}$ are firmly non-expansive. As a result, $M$ is firmly non-expansive [20, Proposition 4.21(i)-(ii)], and equivalently that $M_{\lambda}$ is $\frac{\lambda}{2}$-averaged by Lemma 2.1(i) $\Leftrightarrow$ (iv).

Under the assumptions of Theorem 5.1, there exists $K \in \mathbb{N}$ large enough such that for all $k \geq K,\left(x_{k}, v_{k}\right) \in \mathcal{M}_{x^{\star}}^{J} \times \mathcal{M}_{x^{\star}}^{G}$. Denote $T_{x_{k}}^{J}$ and $T_{x^{\star}}^{J}$ be the tangent spaces corresponding to $x_{k}$ and $x^{\star} \in \mathcal{M}_{x^{\star}}^{J}$, and similarly $T_{x_{k}}^{G}$ and $T_{x^{\star}}^{G}$ the tangent spaces corresponding to $v_{k}$ and $x^{\star} \in \mathcal{M}_{x^{\star}}^{G}$. Denote $\tau_{k}^{J}: T_{x_{k}}^{J} \rightarrow T_{x^{\star}}^{J}\left(\right.$ resp. $\tau_{k}^{G}: T_{v_{k}}^{G} \rightarrow T_{x^{\star}}^{G}$ ) the parallel translation along the unique geodesic on $\mathcal{M}_{x^{\star}}^{J}$ (resp. $\mathcal{M}_{x^{\star}}^{G}$ ) joining $x_{k}$ to $x^{\star}$ (resp. $v_{k}$ to $x^{\star}$ ).

From (6), for $x_{k}$, we have

$$
\left\{\begin{array} { l } 
{ x _ { k } = \operatorname { p r o x } _ { \gamma _ { k } J } ( z _ { k } ) , } \\
{ x ^ { \star } = \operatorname { p r o x } _ { \gamma } ( z ^ { \star } ) , }
\end{array} \Longleftrightarrow \left\{\begin{array}{l}
z_{k}-x_{k} \in \gamma_{k} \partial J\left(x_{k}\right), \\
z^{\star}-x^{\star} \in \gamma \partial J\left(x^{\star}\right) .
\end{array}\right.\right.
$$


Projecting on the corresponding tangent spaces, using Lemma 5.1, and applying the parallel translation operator $\tau_{k}^{J}$ leads to

$$
\begin{aligned}
\gamma_{k} \tau_{k}^{J} \nabla_{\mathcal{M}_{x^{\star}}^{J}} J\left(x_{k}\right) & =\tau_{k}^{J} \mathrm{P}_{T_{x_{k}}^{J}}\left(z_{k}-x_{k}\right)=\mathrm{P}_{T_{x^{\star}}^{J}}\left(z_{k}-x_{k}\right)+\left(\tau_{k}^{J} \mathrm{P}_{T_{x_{k}}^{J}}-\mathrm{P}_{T_{x^{\star}}^{J}}\right)\left(z_{k}-x_{k}\right), \\
\gamma \nabla_{\mathcal{M}_{x^{\star}}^{J}} J\left(x^{\star}\right) & =\mathrm{P}_{T_{x^{\star}}^{J}}\left(z^{\star}-x^{\star}\right) .
\end{aligned}
$$

We then obtain

$$
\begin{aligned}
& \gamma_{k} \tau_{k}^{J} \nabla_{\mathcal{M}_{x^{\star}}^{J}} J\left(x_{k}\right)-\gamma \nabla_{\mathcal{M}_{x^{\star}}^{J}} J\left(x^{\star}\right)=\gamma \tau_{k}^{J} \nabla_{\mathcal{M}_{x^{\star}}^{J}} J\left(x_{k}\right)-\gamma \nabla_{\mathcal{M}_{x^{\star}}^{J}} J\left(x^{\star}\right) \\
& +\left(\gamma_{k}-\gamma\right) \tau_{k}^{J} \nabla_{\mathcal{M}_{x^{\star}}^{J}} J\left(x_{k}\right) \\
& =\mathrm{P}_{T_{x^{\star}}^{J}}\left(\left(z_{k}-z^{\star}\right)-\left(x_{k}-x^{\star}\right)\right) \\
& +\underbrace{\left(\tau_{k}^{J} \mathrm{P}_{T_{x_{k}}^{J}}-\mathrm{P}_{T_{x^{\star}}^{J}}\right)\left(z_{k}-x_{k}-z^{\star}+x^{\star}\right)}_{\text {Term } 1} \\
& +\underbrace{\left(\tau_{k}^{J} \mathrm{P}_{T_{x_{k}}^{J}}-\mathrm{P}_{T_{x^{\star}}^{J}}\right)\left(z^{\star}-x^{\star}\right)}_{\text {Term } 2} .
\end{aligned}
$$

For $\left(\gamma_{k}-\gamma\right) \tau_{k}^{J} \nabla_{\mathcal{M}_{x^{\star}}^{J}} J\left(x_{k}\right)$, since the Riemannian gradient $\nabla_{\mathcal{M}_{x^{\star}}^{J}} J\left(x_{k}\right)$ is single-valued and bounded on bounded sets, we have

$$
\left\|\left(\gamma_{k}-\gamma\right) \tau_{k}^{J} \nabla_{\mathcal{M}_{x^{\star}}^{J}} J\left(x_{k}\right)\right\|=O\left(\left|\gamma_{k}-\gamma\right|\right) .
$$

Combining (24) and (31), we have for Term 1

$$
\left(\tau_{k}^{J} \mathrm{P}_{T_{x_{k}}^{J}}-\mathrm{P}_{T_{x^{\star}}^{J}}\right)\left(z_{k}-x_{k}-z^{\star}+x^{\star}\right)=o\left(z_{k}-z^{\star}\right)+o\left(\left|\gamma_{k}-\gamma\right|\right) .
$$

As far as Term 2 is concerned, with (13), (24) and the Riemannian Taylor expansion (32), we have

$$
\begin{aligned}
& \gamma \tau_{k}^{J} \nabla_{\mathcal{M}_{x^{\star}}^{J}} J\left(x_{k}\right)-\gamma \nabla_{\mathcal{M}_{x^{\star}}^{J}} J\left(x^{\star}\right)-\left(\tau_{k}^{J} \mathrm{P}_{T_{x_{k}}^{J}}-\mathrm{P}_{T_{x^{\star}}^{J}}\right)\left(z^{\star}-x^{\star}\right) \\
& =\tau_{k}^{J}\left(\gamma \nabla_{\mathcal{M}_{x^{\star}}^{J}} J\left(x_{k}\right)-\mathrm{P}_{T_{x_{k}}^{J}}\left(z^{\star}-x^{\star}\right)\right)-\left(\gamma \nabla_{\mathcal{M}_{x^{\star}}^{J}} J\left(x^{\star}\right)-\mathrm{P}_{T_{x^{\star}}^{J}}\left(z^{\star}-x^{\star}\right)\right) \\
& =\tau_{k}^{J} \nabla_{\mathcal{M}_{x^{\star}}^{J}} \bar{J}\left(x_{k}\right)-\nabla_{\mathcal{M}_{x^{\star}}^{J}} \bar{J}\left(x^{\star}\right)=\mathrm{P}_{T_{x^{\star}}^{J}} \nabla_{\mathcal{M}_{x^{\star}}^{J}}^{2} \bar{J}\left(x^{\star}\right) \mathrm{P}_{T_{x^{\star}}^{J}}\left(x_{k}-x^{\star}\right)+o\left(x_{k}-x^{\star}\right) \\
& =\mathrm{P}_{T_{x^{\star}}^{J} \nabla_{\mathcal{M}_{x^{\star}}^{J}}^{2}} \bar{J}\left(x^{\star}\right) \mathrm{P}_{T_{x^{\star}}^{J}}\left(x_{k}-x^{\star}\right)+o\left(z_{k}-z^{\star}\right)+o\left(\left|\gamma_{k}-\gamma\right|\right) .
\end{aligned}
$$

Therefore, inserting (34), (35) and (36) into (33), we obtain

$$
\begin{array}{rlrl}
H_{\bar{J}}\left(x_{k}-x^{\star}\right) & =\mathrm{P}_{T_{x^{\star}}^{J}}\left(z_{k}-z^{\star}\right)-\mathrm{P}_{T_{x^{\star}}^{J}}\left(x_{k}-x^{\star}\right)+o\left(z_{k}-z^{\star}\right)+O\left(\left|\gamma_{k}-\gamma\right|\right) \\
\Rightarrow & & \left(\mathrm{Id}+H_{\bar{J}}\right) \mathrm{P}_{T_{x^{\star}}^{J}}\left(x_{k}-x^{\star}\right) & =\mathrm{P}_{T_{x^{\star}}^{J}}\left(z_{k}-z^{\star}\right)+o\left(z_{k}-z^{\star}\right)+O\left(\left|\gamma_{k}-\gamma\right|\right) \\
\Rightarrow & \mathrm{P}_{T_{x^{\star}}^{J}}\left(x_{k}-x^{\star}\right) & =W_{\bar{J}} \mathrm{P}_{T_{x^{\star}}^{J}}\left(z_{k}-z^{\star}\right)+o\left(z_{k}-z^{\star}\right)+O\left(\left|\gamma_{k}-\gamma\right|\right) \\
\Rightarrow & \mathrm{P}_{T_{x^{\star}}^{J}}\left(x_{k}-x^{\star}\right) & =\mathrm{P}_{T_{x^{\star}}^{J}} W_{\bar{J}} \mathrm{P}_{T_{x^{\star}}^{J}}\left(z_{k}-z^{\star}\right)+o\left(z_{k}-z^{\star}\right)+O\left(\left|\gamma_{k}-\gamma\right|\right) \\
x_{k}-x^{\star} & =M_{\bar{J}}\left(z_{k}-z^{\star}\right)+o\left(z_{k}-z^{\star}\right)+O\left(\left|\gamma_{k}-\gamma\right|\right),
\end{array}
$$

where we used the fact that $x_{k}-x^{\star}=\mathrm{P}_{T_{x^{\star}}^{J}}\left(x_{k}-x^{\star}\right)+o\left(x_{k}-x^{\star}\right)$ [47, Lemma 5.1].

Similarly for $v_{k+1}$, we have

$$
\left\{\begin{array} { r l } 
{ v _ { k + 1 } } & { = \operatorname { p r o x } _ { \gamma _ { k } G } ( 2 x _ { k } - z _ { k } ) , } \\
{ x ^ { \star } } & { = \operatorname { p r o x } _ { \gamma G } ( 2 x ^ { \star } - z ^ { \star } ) , }
\end{array} \Longleftrightarrow \left\{\begin{array}{c}
2 x_{k}-z_{k}-v_{k+1} \in \gamma \partial J\left(v_{k+1}\right), \\
2 x^{\star}-z^{\star}-x^{\star} \in \gamma \partial J\left(x^{\star}\right) .
\end{array}\right.\right.
$$


Upon projecting onto the corresponding tangent spaces and applying the parallel translation $\tau_{k+1}^{G}$, we get

$$
\begin{aligned}
\gamma_{k} \tau_{k+1}^{G} \nabla_{\mathcal{M}_{x^{\star}}^{G}} G\left(v_{k+1}\right) & =\tau_{k+1}^{G} \mathrm{P}_{T_{v_{k+1}}^{G}}\left(2 x_{k}-z_{k}-v_{k+1}\right) \\
& =\mathrm{P}_{T_{x^{\star}}^{G}}\left(2 x_{k}-z_{k}-v_{k+1}\right)+\left(\tau_{k+1}^{G} \mathrm{P}_{T_{v_{k+1}}^{G}}-\mathrm{P}_{T_{x^{\star}}^{G}}\right)\left(2 x_{k}-z_{k}-v_{k+1}\right), \\
\gamma \nabla_{\mathcal{M}_{x^{\star}}^{G}} G\left(x^{\star}\right) & =\mathrm{P}_{T_{x^{\star}}^{G}}\left(2 x^{\star}-z^{\star}-x^{\star}\right) .
\end{aligned}
$$

Substracting both equations, we obtain

$$
\begin{aligned}
& \gamma \tau_{k+1}^{G} \nabla_{\mathcal{M}_{x^{\star}}^{G}} G\left(v_{k+1}\right)-\gamma \nabla_{\mathcal{M}_{x^{\star}}^{G}} G\left(x^{\star}\right)=\gamma \tau_{k+1}^{G} \nabla_{\mathcal{M}_{x^{\star}}^{G}} G\left(v_{k+1}\right)-\gamma \nabla_{\mathcal{M}_{x^{\star}}^{G}} G\left(x^{\star}\right) \\
& +\left(\gamma_{k}-\gamma\right) \tau_{k+1}^{G} \nabla_{\mathcal{M}_{x^{\star}}} G\left(v_{k+1}\right) \\
& =\mathrm{P}_{T_{x^{\star}}^{G}}\left(\left(2 x_{k}-z_{k}-v_{k+1}\right)-\left(2 x^{\star}-z^{\star}-x^{\star}\right)\right) \\
& +\underbrace{\left(\tau_{k+1}^{G} \mathrm{P}_{T_{v_{k+1}}^{G}}-\mathrm{P}_{T_{x^{\star}}^{G}}\right)\left(x^{\star}-z^{\star}\right)}_{\text {Term } 4} \\
& +\underbrace{\left(\tau_{k+1}^{G} \mathrm{P}_{T_{v_{k+1}}^{G}}-\mathrm{P}_{T_{x^{\star}}}\right)\left(\left(2 x_{k}-z_{k}-v_{k+1}\right)-\left(2 x^{\star}-z^{\star}-x^{\star}\right)\right)}_{\text {Term } 3} .
\end{aligned}
$$

As for (34), we have

$$
\left\|\left(\gamma_{k}-\gamma\right) \tau_{k+1}^{G} \nabla_{\mathcal{M}_{x^{\star}}^{G}} G\left(v_{k+1}\right)\right\|=O\left(\left|\gamma_{k}-\gamma\right|\right) .
$$

With similar arguments to those used for Term 1, we have Term $3=o\left(z_{k}-z^{\star}\right)+o\left(\left|\gamma_{k}-\gamma\right|\right)$. Moreover, similarly to (36), we have for Term 4,

$$
\begin{aligned}
& \gamma \tau_{k+1}^{G} \nabla_{\mathcal{M}_{x^{\star}}^{G}} G\left(v_{k+1}\right)-\gamma \nabla_{\mathcal{M}_{x^{\star}}^{G}} G\left(x^{\star}\right)-\left(\tau_{k+1}^{G} \mathrm{P}_{T_{v_{k+1}}^{G}}-\mathrm{P}_{T_{x^{\star}}^{G}}\right)\left(x^{\star}-z^{\star}\right) \\
& =\mathrm{P}_{T_{x^{\star}}^{G}} \nabla_{\mathcal{M}_{x^{\star}}^{G}}^{2} \bar{G}\left(x^{\star}\right) \mathrm{P}_{T_{x^{\star}}^{G}}\left(v_{k+1}-x^{\star}\right)+o\left(z_{k}-x^{\star}\right)+o\left(\left|\gamma_{k}-\gamma\right|\right) .
\end{aligned}
$$

Then for (38) we have,

$$
\begin{aligned}
H_{\bar{G}}\left(v_{k+1}-x^{\star}\right)= & 2 \mathrm{P}_{T_{x^{\star}}^{G}}\left(x_{k}-x^{\star}\right)-\mathrm{P}_{T_{x^{\star}}^{G}}\left(z_{k}-z^{\star}\right)-\mathrm{P}_{T_{x^{\star}}}\left(v_{k+1}-x^{\star}\right) \\
& +o\left(z_{k}-z^{\star}\right)+O\left(\left|\gamma_{k}-\gamma\right|\right) \\
\Rightarrow \quad\left(\mathrm{Id}+H_{\bar{G}}\right) \mathrm{P}_{T_{x^{\star}}}\left(v_{k+1}-x^{\star}\right)= & \mathrm{P}_{T_{x^{\star}}^{G}}\left(x_{k}-x^{\star}\right)-\mathrm{P}_{T_{x^{\star}}}\left(z_{k}-z^{\star}\right) \\
& +o\left(z_{k}-z^{\star}\right)+O\left(\left|\gamma_{k}-\gamma\right|\right) \\
\mathrm{P}_{T_{x^{\star}}^{G}}\left(v_{k+1}-x^{\star}\right)=2 & M_{\bar{G}} M_{\bar{J}}\left(z_{k}-z^{\star}\right)-M_{\bar{G}}\left(z_{k}-z^{\star}\right) \\
& +o\left(z_{k}-z^{\star}\right)+O\left(\left|\gamma_{k}-\gamma\right|\right) \\
v_{k+1}-x^{\star}=2 & M_{\bar{G}} M_{\bar{J}}\left(z_{k}-z^{\star}\right)-M_{\bar{G}}\left(z_{k}-z^{\star}\right) \\
& +o\left(z_{k}-z^{\star}\right)+O\left(\left|\gamma_{k}-\gamma\right|\right)
\end{aligned}
$$

where $v_{k+1}-x^{\star}=\mathrm{P}_{T_{x^{\star}}^{G}}\left(v_{k+1}-x^{\star}\right)+o\left(v_{k+1}-x^{\star}\right)$ is applied again [47, Lemma 5.1].

Summing up (37) and (41), we get

$$
\begin{aligned}
\left(z_{k}+v_{k+1}-x_{k}\right)-\left(z^{\star}+x^{\star}-x^{\star}\right)= & \left(z_{k}-z^{\star}\right)+\left(v_{k+1}-x^{\star}\right)-\left(x_{k}-x^{\star}\right) \\
= & \left(\operatorname{Id}+2 M_{\bar{G}} M_{\bar{J}}-M_{\bar{G}}-M_{\bar{J}}\right)\left(z_{k}-z^{\star}\right) \\
& \quad+o\left(z_{k}-z^{\star}\right)+O\left(\left|\gamma_{k}-\gamma\right|\right) \\
= & M\left(z_{k}-z^{\star}\right)+o\left(z_{k}-z^{\star}\right)+O\left(\left|\gamma_{k}-\gamma\right|\right) .
\end{aligned}
$$

Hence for the relaxed DR iteration, we have

$$
\begin{aligned}
z_{k+1}-z^{\star} & =\left(1-\lambda_{k}\right)\left(z_{k}-z^{\star}\right)+\lambda_{k}\left(\left(z_{k}+v_{k+1}-x_{k}\right)-\left(z^{\star}+x^{\star}-x^{\star}\right)\right) \\
& =\left(1-\lambda_{k}\right)\left(z_{k}-z^{\star}\right)+\lambda_{k} M\left(z_{k}-z^{\star}\right)+o\left(z_{k}-z^{\star}\right)+\phi_{k} \\
& =M_{\lambda}\left(z_{k}-z^{\star}\right)-\left(\lambda_{k}-\lambda\right)(\operatorname{Id}-M)\left(z_{k}-z^{\star}\right)+o\left(z_{k}-z^{\star}\right)+\phi_{k}
\end{aligned}
$$


Since Id $-M$ is also (firmly) non-expansive (Lemma 2.1(ii)) and $\left.\lambda_{k} \rightarrow \lambda \in\right] 0,2[$, we thus get

$\lim _{k \rightarrow \infty} \frac{\left\|\left(\lambda_{k}-\lambda\right)(\operatorname{Id}-M)\left(z_{k}-z^{\star}\right)\right\|}{\left\|z_{k}-z^{\star}\right\|}=\lim _{k \rightarrow \infty} \frac{\left|\lambda_{k}-\lambda\right|\left\|(\operatorname{Id}-M)\left(z_{k}-z^{\star}\right)\right\|}{\left\|z_{k}-z^{\star}\right\|} \leq \lim _{k \rightarrow \infty}\left|\lambda_{k}-\lambda\right|=0$,

which means that

$$
z_{k+1}-z^{\star}=M_{\lambda}\left(z_{k}-z^{\star}\right)+\psi_{k}+\phi_{k},
$$

and the claimed result is obtained.

Proof (Lemma 6.2)

(i) Since $M$ is firmly non-expansive and $M_{\lambda}$ is $\frac{\lambda}{2}$-averaged by Proposition 6.1, we deduce from [20, Proposition 5.15] that $M$ and $M_{\lambda}$ are convergent, and their limit is $M_{\lambda}^{\infty}=\mathrm{P}_{\mathrm{Fix}\left(M_{\lambda}\right)}=\mathrm{P}_{\mathrm{Fix}(M)}=$ $M^{\infty}$ [22, Corollary 2.7(ii)]. Moreover, $M_{\lambda}^{k}-M^{\infty}=\left(M_{\lambda}-M^{\infty}\right)^{k}, \forall k \in \mathbb{N}$, and $\rho\left(M_{\lambda}-\right.$ $\left.M^{\infty}\right)<1$ by [22, Theorem 2.12]. It is also immediate to see that

$$
\operatorname{Fix}(M)=\operatorname{ker}\left(M_{\bar{G}}\left(\operatorname{Id}-M_{\bar{J}}\right)+\left(\operatorname{Id}-M_{\bar{G}}\right) M_{\bar{J}}\right) .
$$

Observe that

$$
\begin{gathered}
\operatorname{span}\left(M_{\bar{J}}\right) \subseteq T_{x^{\star}}^{J} \text { and } \operatorname{span}\left(M_{\bar{G}}\right) \subseteq T_{x^{\star}}^{G}, \\
\operatorname{ker}\left(\operatorname{Id}-M_{\bar{G}}\right) \subseteq T_{x^{\star}}^{G} \text { and } \operatorname{ker}\left(M_{\bar{G}}\right)=S_{x^{\star}}^{G}, \\
\operatorname{span}\left(\left(\operatorname{Id}-M_{\bar{G}}\right) M_{\bar{J}}\right) \subseteq \operatorname{span}\left(\operatorname{Id}-M_{\bar{G}}\right) \text { and } \operatorname{span}\left(M_{\bar{G}}\left(\operatorname{Id}-M_{\bar{J}}\right)\right) \subseteq T_{x^{\star}}^{G},
\end{gathered}
$$

where we used the fact that $W_{\bar{G}}$ and $W_{\bar{J}}$ are positive definite. Therefore, $M_{\lambda}^{\infty}=0$, if and only if, $\operatorname{Fix}(M)=\{0\}$, and for this to hold true, it is sufficient that

$$
\begin{aligned}
& \operatorname{span}\left(M_{\bar{J}}\right) \cap \operatorname{ker}\left(\operatorname{Id}-M_{\bar{G}}\right) \subseteq T_{x^{\star}}^{J} \cap T_{x^{\star}}^{G}=\{0\}, \\
& \operatorname{span}\left(\operatorname{Id}-M_{\bar{J}}\right) \cap \operatorname{ker}\left(M_{\bar{G}}\right)=\operatorname{span}\left(\operatorname{Id}-M_{\bar{J}}\right) \cap S_{x^{\star}}^{G}=\{0\}, \\
& \operatorname{span}\left(\left(\operatorname{Id}-M_{\bar{G}}\right) M_{\bar{J}}\right) \cap \operatorname{span}\left(M_{\bar{G}}\left(\operatorname{Id}-M_{\bar{J}}\right)\right) \subseteq \operatorname{span}\left(\operatorname{Id}-M_{\bar{G}}\right) \cap T_{x^{\star}}^{G}=\{0\} .
\end{aligned}
$$

(ii) The proof is classical using the spectral radius formula (2), see e.g. [22, Theorem 2.12(i)]

(iii) In this case, we have $W_{\bar{G}}=W_{\bar{J}}=\mathrm{Id}$. In turn, $M_{\bar{G}}=\mathrm{P}_{T_{x^{\star}}^{G}}$ and $M_{\bar{J}}=\mathrm{P}_{T_{x^{\star}}^{J}}$, which yields

$$
M=\mathrm{Id}+2 \mathrm{P}_{T_{x^{\star}}^{G}} \mathrm{P}_{T_{x^{\star}}^{J}}-\mathrm{P}_{T_{x^{\star}}^{G}}-\mathrm{P}_{T_{x^{\star}}^{J}}=\mathrm{P}_{T_{x^{\star}}^{G}} \mathrm{P}_{T_{x^{\star}}^{J}}+\mathrm{P}_{S_{x^{\star}}^{G}} \mathrm{P}_{S_{x^{\star}}^{J}},
$$

which is normal, and so is $M_{\lambda}$. From [12, Proposition 3.6(i)], we get that $\operatorname{Fix}(M)=\left(T_{x^{\star}}^{J} \cap T_{x^{\star}}^{G}\right) \oplus\left(S_{x^{\star}}^{J} \cap S_{x^{\star}}^{G}\right)$. Thus, combining normality, statement (i) and [22, Theorem 2.16] we get that

$$
\left\|M_{\lambda}^{k+1-K}-M^{\infty}\right\|=\left\|M_{\lambda}-M^{\infty}\right\|^{k+1-K},
$$

and $\left\|M_{\lambda}-M^{\infty}\right\|$ is the optimal convergence rate of $M_{\lambda}$. Combining together [22, Proposition 3.3] and arguments similar to those of the proof of [12, Theorem 3.10(ii)] (see also [22, Theorem 4.1(ii)]), we get indeed that

$$
\left\|M_{\lambda}-M^{\infty}\right\|=\sqrt{(1-\lambda)^{2}+\lambda(2-\lambda) \cos ^{2}\left(\theta_{F}\left(T_{x^{\star}}^{J}, T_{x^{\star}}^{G}\right)\right)} .
$$

The special case is immediate. This concludes the proof

Proof (Corollary 6.1)

(i) Let $K \in \mathbb{N}$ sufficiently large such that the locally linearized iteration (17) holds. Then we have for $k \geq K$

$$
\begin{aligned}
z_{k+1}-z^{\star} & =M_{\lambda}\left(z_{k}-z^{\star}\right)+\psi_{k}+\phi_{k}=M_{\lambda}\left(M_{\lambda}\left(z_{k-1}-z^{\star}\right)+\psi_{k-1}+\phi_{k-1}\right)+\psi_{k}+\phi_{k} \\
& =M_{\lambda}^{k+1-K}\left(z_{K}-z^{\star}\right)+\sum_{j=K}^{k} M_{\lambda}^{k-j}\left(\psi_{j}+\phi_{j}\right) .
\end{aligned}
$$


Since $z_{k} \rightarrow z^{\star}$ from Theorem 4.1 and $M_{\lambda}$ is convergent to $M^{\infty}$ by Lemma 6.2(i), taking the limit as $k \rightarrow \infty$, we have for all finite $p \geq K$,

$$
\lim _{k \rightarrow \infty} \sum_{j=p}^{k} M_{\lambda}^{k-j}\left(\psi_{j}+\phi_{j}\right)=-M^{\infty}\left(z_{p}-z^{\star}\right) .
$$

Using (43) in (42), we get

$$
\begin{aligned}
z_{k+1}-z^{\star}=\left(M_{\lambda}-M^{\infty}\right)\left(z_{k}-z^{\star}\right)+\psi_{k}+\phi_{k}-\lim _{l \rightarrow \infty} \sum_{j=k}^{l} M_{\lambda}^{l-j}\left(\psi_{j}+\phi_{j}\right) \\
=\left(M_{\lambda}-M^{\infty}\right)\left(z_{k}-z^{\star}\right)+\psi_{k}+\phi_{k}-\lim _{l \rightarrow \infty} \sum_{j=k+1}^{l} M_{\lambda}^{l-j}\left(\psi_{j}+\phi_{j}\right) \\
-M^{\infty}\left(\psi_{k}+\phi_{k}\right) \\
=\left(M_{\lambda}-M^{\infty}\right)\left(z_{k}-z^{\star}\right)+\left(\mathrm{Id}-M^{\infty}\right)\left(\psi_{j}+\phi_{j}\right)+M^{\infty}\left(z_{k+1}-z^{\star}\right) .
\end{aligned}
$$

It is also immediate to see from Lemma 6.2(i) that $\left\|\mathrm{Id}-M^{\infty}\right\| \leq 1$ and

$$
\left(M_{\lambda}-M^{\infty}\right)\left(\mathrm{Id}-M^{\infty}\right)=M_{\lambda}-M^{\infty} .
$$

Rearranging the terms gives the claimed equivalence.

(ii) Under polyhedrality and constant parameters, we have from Proposition 6.1 that both $\phi_{k}$ and $\psi_{k}$ vanish. In this case, (43) reads

$$
z_{k}-z^{\star} \in \operatorname{ker}\left(M^{\infty}\right), \quad \forall k \geq K
$$

and therefore (17) obviously becomes (19).

Proof (Theorem 6.1)

(i) Let $K \in \mathbb{N}$ sufficiently large such that (18) holds. We then have from Corollary 6.1(i)

$$
\begin{aligned}
\left(\operatorname{Id}-M^{\infty}\right)\left(z_{k+1}-z^{\star}\right)=( & \left.M_{\lambda}-M^{\infty}\right)^{k+1-K}\left(\operatorname{Id}-M^{\infty}\right)\left(z_{K}-z^{\star}\right) \\
& +\sum_{j=K}^{k}\left(M_{\lambda}-M^{\infty}\right)^{k-j}\left(\left(\operatorname{Id}-M^{\infty}\right) \psi_{j}+\phi_{j}\right) .
\end{aligned}
$$

Since $\rho\left(M_{\lambda}-M^{\infty}\right)<1$ by Lemma 6.2(i), from the spectral radius formula, we know that for every $\rho \in] \rho\left(M_{\lambda}-M^{\infty}\right), 1[$, there is a constant $C$ such that

$$
\left\|\left(M_{\lambda}-M^{\infty}\right)^{j}\right\| \leq C \rho^{j}
$$

for all integers $j$. We thus get

$$
\begin{aligned}
\left\|\left(\operatorname{Id}-M^{\infty}\right)\left(z_{k+1}-z^{\star}\right)\right\| \leq & C \rho^{k+1-K}\left\|z_{K}-z^{\star}\right\|+C \sum_{j=K}^{k} \rho^{k-j} \phi_{j} \\
& +C \sum_{j=K}^{k} \rho^{k-j}\left\|\left(\operatorname{Id}-M^{\infty}\right) \psi_{j}\right\| \\
= & C \rho^{k+1-K}\left(\left\|z_{K}-z^{\star}\right\|+\rho^{K-1} \sum_{j=K}^{k} \frac{\phi_{j}}{\rho^{j}}\right) \\
& +C \sum_{j=K}^{k} \rho^{k-j}\left\|\left(\mathrm{Id}-M^{\infty}\right) \psi_{j}\right\|,
\end{aligned}
$$

By assumption, $\phi_{j}=C^{\prime} \eta^{j}$, for some constant $C^{\prime} \geq 0$ and $\eta<\rho$, and we have

$$
\rho^{K-1} \sum_{j=K}^{k} \frac{\phi_{j}}{\rho^{j}} \leq C^{\prime} \rho^{K-1} \sum_{j=K}^{\infty}(\eta / \rho)^{j}=\frac{C^{\prime} \eta^{K}}{\rho-\eta}<+\infty .
$$

Setting $C^{\prime \prime}=C\left(\left\|z_{K}-z^{\star}\right\|+\frac{C^{\prime} \eta^{K}}{\rho-\eta}\right)<+\infty$, we obtain

$$
\left\|\left(\operatorname{Id}-M^{\infty}\right)\left(z_{k+1}-z^{\star}\right)\right\| \leq C^{\prime \prime} \rho^{k+1-K}+C \sum_{j=K}^{k} \rho^{k-j}\left\|\left(\operatorname{Id}-M^{\infty}\right) \psi_{j}\right\| .
$$

This, together with the fact that $\left\|\left(\operatorname{Id}-M^{\infty}\right) \psi_{j}\right\|=o\left(\left\|\left(\operatorname{Id}-M^{\infty}\right)\left(z_{j}-z^{\star}\right)\right\|\right)$ yields the claimed result. 
(ii) From Corollary 6.1(ii), we have

$$
z_{k}-z^{\star}=\left(M_{\lambda}-M^{\infty}\right)^{k+1-K}\left(z_{K}-z^{\star}\right) .
$$

Moreover, by virtue of Lemma 6.2(iii), $M_{\lambda}$ is normal and converges linearly to

$$
M^{\infty}=\mathrm{P}_{\left(T_{x^{\star}}^{J} \cap T_{x^{\star}}^{G}\right) \oplus\left(S_{x^{\star}}^{J} \cap S_{x^{\star}}^{G}\right)}
$$

at the optimal rate

$$
\rho=\left\|M_{\lambda}-M^{\infty}\right\|=\sqrt{(1-\lambda)^{2}+\lambda(2-\lambda) \cos ^{2}\left(\theta_{F}\left(T_{x^{\star}}^{J}, T_{x^{\star}}^{G}\right)\right)} .
$$

Combining all this then entails

$$
\begin{aligned}
\left\|z_{k+1}-z^{\star}\right\| \leq\left\|\left(M_{\lambda}-M^{\infty}\right)^{k+1-K}\right\|\left(z_{K}-z^{\star}\right) & =\left\|M_{\lambda}-M^{\infty}\right\|^{k+1-K}\left\|z_{K}-z^{\star}\right\| \\
& =\rho^{k+1-K}\left\|z_{K}-z^{\star}\right\|,
\end{aligned}
$$

which concludes the proof.

\section{Acknowledgments C}

This work has been partly supported by the European Research Council (ERC project SIGMA-Vision). JF was partly supported by Institut Universitaire de France. The authors would like to thank Russell Luke for helpful discussions.

\section{References C}

1. Douglas, J., Rachford, H.H.: On the numerical solution of heat conduction problems in two and three space variables. Transactions of the American mathematical Society 82(2), 421-439 (1956)

2. Lions, P.L., Mercier, B.: Splitting algorithms for the sum of two nonlinear operators. SIAM Journal on Numerical Analysis 16(6), 964-979 (1979)

3. Liang, J., Fadili, M.J., Peyré, G.: Convergence rates with inexact non-expansive operators. Mathematical Programming pp. 1-32 (2015). DOI 10.1007/s10107-015-0964-4. URL http://dx.doi.org/ 10.1007/s10107-015-0964-4

4. Davis, D., Yin, W.: Convergence rate analysis of several splitting schemes. Tech. rep., arXiv:1406.4834 (2014)

5. Davis, D., Yin, W.: Convergence rates of relaxed Peaceman-Rachford and ADMM under regularity assumptions. Tech. rep., arXiv:1407.5210 (2014)

6. Giselsson, P., Boyd, S.: Metric selection in Douglas-Rachford Splitting and ADMM. arXiv preprint arXiv:1410.8479 (2014)

7. Gabay, D.: Applications of the method of multipliers to variational inequalities. In: M. Fortin, R. Glowinski (eds.) Augmented Lagrangian Methods: Applications to the Solution of Boundary-Value Problems, pp. 299-331. Elsevier, North-Holland, Amsterdam (1983)

8. Eckstein, J., Bertsekas, D.P.: On the Douglas-Rachford splitting method and the proximal point algorithm for maximal monotone operators. Mathematical Programming 55(1-3), 293-318 (1992)

9. Lewis, A.S.: Active sets, nonsmoothness, and sensitivity. SIAM Journal on Optimization 13(3), 702725 (2003)

10. Demanet, L., Zhang, X.: Eventual linear convergence of the Douglas-Rachford iteration for basis pursuit. Mathematics of Computation 85(297), 209-238 (2016)

11. Boley, D.: Local linear convergence of the alternating direction method of multipliers on quadratic or linear programs. SIAM Journal on Optimization 23(4), 2183-2207 (2013)

12. Bauschke, H., Cruz, J., Nghia, T., Phan, H., Wang, X.: The rate of linear convergence of the douglasrachford algorithm for subspaces is the cosine of the Friedrichs angle. J. of Approx. Theo. 185(63-79) (2014) 
13. Liang, J., Fadili, M.J., Peyré, G., Luke, R.: Activity identification and local linear convergence of Douglas-Rachford/ADMM under partial smoothness. In: Scale Space and Variational Methods in Computer Vision, pp. 642-653. Springer (2015)

14. Borwein, J.M., Sims, B.: The Douglas-Rachford algorithm in the absence of convexity. In: H.H. Bauschke, R.S. Burachik, P.L. Combettes, V. Elser, D.R. Luke, H. Wolkowicz (eds.) Fixed-Point Algorithms for Inverse Problems in Science and Engineering, Springer Optimization and Its Applications, vol. 49, pp. 93-109. Springer New York (2011)

15. Lewis, A.S., Luke, D.R., Malick, J.: Local linear convergence for alternating and averaged nonconvex projections. Found. Comput. Math. 9(4), 485-513 (2009)

16. Hesse, R., Luke, D.R., Neumann, P.: Projection methods for sparse affine feasibility: Results and counterexamples. Tech. rep. (2013)

17. Hesse, R., Luke, D.R.: Nonconvex notions of regularity and convergence of fundamental algorithms for feasibility problems. SIAM Journal on Optimization 23(4), 2397-2419 (2013)

18. Phan, H.M.: Linear convergence of the Douglas-Rachford method for two closed sets. Optimization 65(2), 369-385 (2016)

19. Bauschke, H.H., Dao, M.N., Noll, D., Phan, H.M.: On Slater's condition and finite convergence of the Douglas-Rachford algorithm for solving convex feasibility problems in Euclidean spaces. Journal of Global Optimization pp. 1-21 (2015). In press

20. Bauschke, H.H., Combettes, P.L.: Convex analysis and monotone operator theory in Hilbert spaces. Springer (2011)

21. Combettes, P.L., Yamada, I.: Compositions and convex combinations of averaged nonexpansive operators. Journal of Mathematical Analysis and Applications 425(1), 55-70 (2015)

22. Bauschke, H.H., Bello Cruz, J.Y., Nghia, T.T.A., Pha, H.M., Wang, X.: Optimal rates of linear convergence of relaxed alternating projections and generalized Douglas-Rachford methods for two subspaces. Numerical Algorithms 73(1), 33-76 (2016). DOI 10.1007/s11075-015-0085-4. URL http://dx.doi.org/10.1007/s11075-015-0085-4

23. Combettes, P.L.: Fejér monotonicity in convex optimization. In: A.C. Floudas, M.P. Pardalos (eds.) Encyclopedia of Optimization, pp. 1016-1024. Springer, Boston, MA (2001). DOI 10.1007/ 978-0-387-74759-0_179. URL http://dx.doi.org/10.1007/978-0-387-74759-0_179

24. Combettes, P.L.: Solving monotone inclusions via compositions of nonexpansive averaged operators. Optimization 53(5-6), 475-504 (2004)

25. Bauschke, H.H., Moursi, W.: On the order of the operators in the Douglas-Rachford algorithm. Optimization Letters (2016). In press (arXiv:1505.02796v1)

26. Combettes, P.L.: Quasi-Fejérian analysis of some optimization algorithms. Studies in Computational Mathematics 8, 115-152 (2001)

27. Wright, S.J.: Identifiable surfaces in constrained optimization. SIAM Journal on Control and Optimization 31(4), 1063-1079 (1993)

28. Lemaréchal, C., Oustry, F., Sagastizábal, C.: The U-lagrangian of a convex function. Trans. Amer. Math. Soc. 352(2), 711-729 (2000)

29. Daniilidis, A., Drusvyatskiy, D., Lewis, A.S.: Orthogonal invariance and identifiability. SIAM J. Matrix Anal. Appl. 35, 580-598 (2014)

30. Liang, J., Fadili, M.J., Peyré, G.: Activity identification and local linear convergence of ForwardBackward-type methods (2015). Submitted (arXiv:1503.03703)

31. Hare, W., Lewis, A.S.: Identifying active manifolds. Alg. Op. Res. 2(2), 75-82 (2007)

32. Rockafellar, R.T., Wets, R.: Variational analysis, vol. 317. Springer Verlag (1998)

33. Rockafellar, R.T.: Convex analysis, vol. 28. Princeton university press (1997)

34. Kim, N., Luc, D.: Normal cones to a polyhedral convex set and generating efficient faces in multiobjective linear programming. Acta Math. Vietnam. 25, 101-124 (2000)

35. Rockafellar, R.T.: Monotone operators and the proximal point algorithm. SIAM Journal on Control and Optimization 14(5), 877-898 (1976)

36. Luque, F.: Asymptotic convergence analysis of the proximal point algorithm. SIAM Journal on Control and Optimization 22, 277-293 (1984)

37. Combettes, P.L., Pesquet, J.C.: A proximal decomposition method for solving convex variational inverse problems. Inverse Problems 24(6), 065,014 (2008). URL http://stacks.iop.org/ $0266-5611 / 24 / i=6 / a=065014$

38. Raguet, H., Fadili, M.J., Peyré, G.: A generalized Forward-Backward splitting. SIAM Journal on Imaging Sciences 6(3), 1199-1226 (2013) 
39. Vaiter, S., Deledalle, C., Fadili, J.M., Peyré, G., Dossal, C.: The degrees of freedom of partly smooth regularizers. Annals of the Institute of Statistical Mathematics (2015). URL http://arxiv .org/ abs/1404.5557. To appear

40. Vaiter, S., Peyré, G., Fadili, M.J.: Model consistency of partly smooth regularizers. Tech. Rep. arXiv:1307.2342, submitted (2015)

41. Brézis, H.: Opérateurs maximaux monotones et semi-groupes de contractions dans les espaces de Hilbert. North-Holland Math. Stud. Elsevier, New York (1973)

42. Hare, W.L., Lewis, A.S.: Identifying active constraints via partial smoothness and prox-regularity. Journal of Convex Analysis 11(2), 251-266 (2004)

43. Chavel, I.: Riemannian geometry: a modern introduction, vol. 98. Cambridge University Press (2006)

44. Miller, S.A., Malick, J.: Newton methods for nonsmooth convex minimization: connections amongLagrangian, Riemannian Newton and SQP methods. Mathematical programming 104(2-3), 609-633 (2005)

45. Absil, P.A., Mahony, R., Trumpf, J.: An extrinsic look at the Riemannian Hessian. In: Geometric Science of Information, pp. 361-368. Springer (2013)

46. Lee, J.M.: Smooth manifolds. Springer (2003)

47. Liang, J., Fadili, M.J., Peyré, G.: Local linear convergence of Forward-Backward under partial smoothness. In: Advances in Neural Information Processing Systems, pp. 1970-1978 (2014) 